\title{
Optically bright active galactic nuclei in the ROSAT-Faint source catalogue ${ }^{\star}$
}

\author{
M.-P. Véron-Cetty ${ }^{1}$, S. K. Balayan², A. M. Mickaelian² ${ }^{2}$ R. Mujica ${ }^{3}$, V. Chavushyan ${ }^{3}$, S. A. Hakopian² ${ }^{2}$ D. Engels ${ }^{4}$, \\ P. Véron ${ }^{1}$, F.-J. Zickgraf ${ }^{4}$, W. Voges ${ }^{5}$, and D.-W. $\mathrm{Xu}^{6,5}$ \\ 1 Observatoire de Haute Provence, CNRS, 04870 Saint-Michel l'Observatoire, France \\ e-mail: philippe.veron@oamp.fr \\ 2 Byurakan Astrophysical Observatory and Isaac Newton Institute of Chile, Armenian branch, Byurakan 378433, \\ Aragatzotn province, Armenia \\ e-mail: bal@moon.yerphi.am; aregmick@apaven.am; sanna@eugene.yerphi.am \\ 3 INAOE, Apdo. postal 51 y 216, 72000 Puebla, Pue., Mexico \\ e-mail: [rmujica; vahram] @inaoep.mx \\ ${ }^{4}$ Hamburger Sternwarte, Gojenbergsweg 112, 21029 Hamburg, Germany \\ e-mail: [dengels; fzickgraf]@hs.uni-hamburg.de \\ 5 Max-Planck-Institute für extraterrestrische Physik, Postfach 1312, 85741, Garching, Germany \\ e-mail: whv@mpe-garching .mpg.de \\ ${ }^{6}$ National astronomical observatories, Beijing 100012, PR China \\ e-mail: dwxu@bao.ac.cn
}

Received 10 June 2003 / Accepted 17 October 2003

\begin{abstract}
To build a large, optically bright, X-ray selected AGN sample we have correlated the ROSAT-FSC catalogue of X-ray sources with the USNO catalogue limited to objects brighter than $O=16.5$ and then with the APS database. Each of the 3212 coincidences was classified using the slitless Hamburg spectra. 493 objects were found to be extended and 2719 starlike. Using both the extended objects and the galaxies known from published catalogues we built up a sample of 185 galaxies with $O_{\mathrm{APS}}<17.0 \mathrm{mag}$, which are high-probability counterparts of RASS-FSC X-ray sources. 130 galaxies have a redshift from the literature and for another 34 we obtained new spectra. The fraction of Seyfert galaxies in this sample is $20 \%$. To select a corresponding sample of 144 high-probability counterparts among the starlike sources we searched for very blue objects in an APS-based color-magnitude diagram. Forty-one were already known AGN and for another 91 objects we obtained new spectra, yielding 42 new AGN, increasing their number in the sample to 83. This confirms that surveys of bright QSOs are still significantly incomplete. On the other hand we find that, at a flux limit of 0.02 count s$^{-1}$ and at this magnitude, only $40 \%$ of all QSOs are detected by ROSAT.
\end{abstract}

Key words. quasars: general - X-rays: galaxies - galaxies: Seyfert

\section{Introduction}

The ROSAT All-Sky Survey Bright Source Catalogue (RASSBSC) is derived from the all-sky survey performed during the ROSAT mission in the energy band $0.1-2.4 \mathrm{keV}$; it contains 18811 sources down to a limiting ROSAT-PSPC count-rate of 0.05 count s$^{-1}$ (Voges et al. 1999). The 2012 brightest (countrate above 0.2 count $\mathrm{s}^{-1}$ ) high galactic latitude $\left(|b|>30^{\circ}\right)$ sources of the BSC catalogue have been tentatively optically identified by Schwope et al. (2000). The ROSAT Faint Source

Send offprint requests to: M.-P. Véron-Cetty, e-mail: mira.veron@oamp.fr

* Tables 2, 3 and 4 are only available in electronic form at http://www. edpsciences.org
Catalogue (FSC) (Voges et al. 2000) contains 105924 sources and represents the faint extension of the RASS-BSC.

The Hamburg QSO Survey is a wide-angle objective prism survey for finding bright QSOs in the northern sky. The survey plates have been taken with the former Hamburg Schmidt telescope now located at the Spanish-German Center in Calar Alto (Spain). A 1.7 objective prism has been used providing unwidened spectra with a dispersion of $1390 \AA \mathrm{mm}^{-1}$ at $\mathrm{H} \gamma$ (Hagen et al. 1995). The slitless spectra allow the classification of objects brighter than about $B=17.0$. The first study of a sample of previously known QSOs with the Einstein Observatory has shown that, as a class, they are luminous X-ray emitters (Tananbaum et al. 1979). Bade et al. (1998) and Zickgraf et al. (2003) have described a way to identify X-ray sources by using the Hamburg survey plates; this 


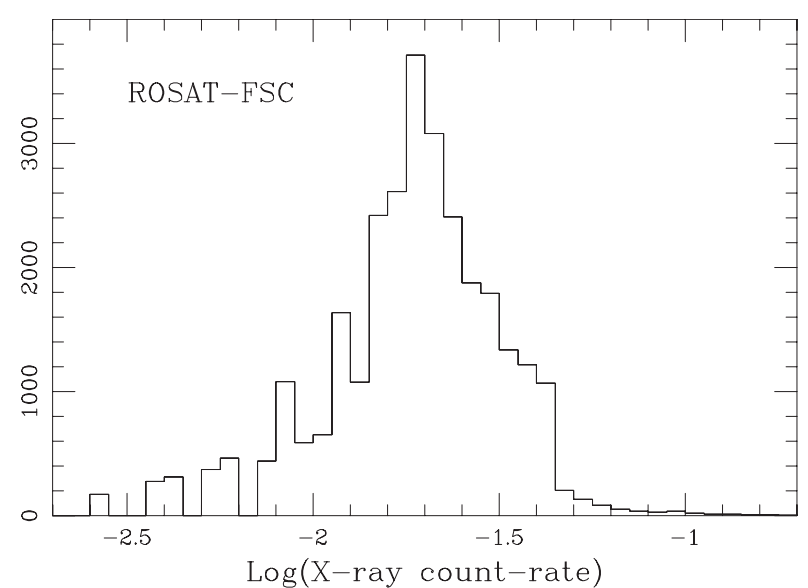

Fig. 1. Histogram of the logarithm of the X-ray count rates (in counts s${ }^{-1}$ ) of the 29321 ROSAT-FSC sources.

process has been applied to a subsample of 5341 sources from the ROSAT-BSC.

In the present paper we try to find all AGN brighter than $O=16.5$ in a subarea of the ROSAT-FSC. As the FSC survey is only about 2.5 times deeper than the BSC survey, we do not expect a significant difference in the identification content of these two samples but, because the number of sources in the FSC survey is much larger, we expect the discovery of many additional optically bright AGN. As the ROSAT survey is a low-energy survey, it contains a relatively high fraction of Narrow-Line Seyfert 1 Galaxies (NLS1s) because they usually have a soft X-ray excess (see for instance Laor et al. 1997); we therefore expect to find new bright NLS1s.

In the following we call AGN (or Active Galactic Nuclei) QSOs, BL Lac objects, Seyfert galaxies and Liners. QSOs are defined as Seyfert 1 galaxies brighter than $M_{B}=-23 \cdot 0^{1}$.

\section{Analysis}

\subsection{The sample}

In the area of the sky defined by $\delta_{1950}>0^{\circ}$ and $|b|>30^{\circ}$ $\left(10313 \mathrm{deg}^{2}\right)$ there are 29321 ROSAT-FSC sources.

Figure 1 shows the histogram of the logarithm of the $\mathrm{X}$ ray count rates of these sources (in counts $\mathrm{s}^{-1}$ ). Below 0.02 counts $\mathrm{s}^{-1}$ the survey is obviously quite incomplete, reflecting the fact that its sensitivity limit is not uniform over the sky. On the other hand 130 sources have a count rate greater than 0.05 counts $\mathrm{s}^{-1}$ but were not included in the BSC as their detected photon number was less than 15 . They were included in the FSC.

Figure 2 shows the distribution over the sky of (a) the 29321 sources and (b) the 15848 sources with $F_{x}>$ 0.02 counts $\mathrm{s}^{-1}$. The second distribution is much more uniform than the first and, in the following, we shall restrict our analysis to this subsample.

\footnotetext{
1 Throughout this work we used $H_{\mathrm{o}}=50 \mathrm{~km} \mathrm{~s}^{-1} \mathrm{Mpc}^{-1}$ and $q_{\mathrm{o}}=0$.
}

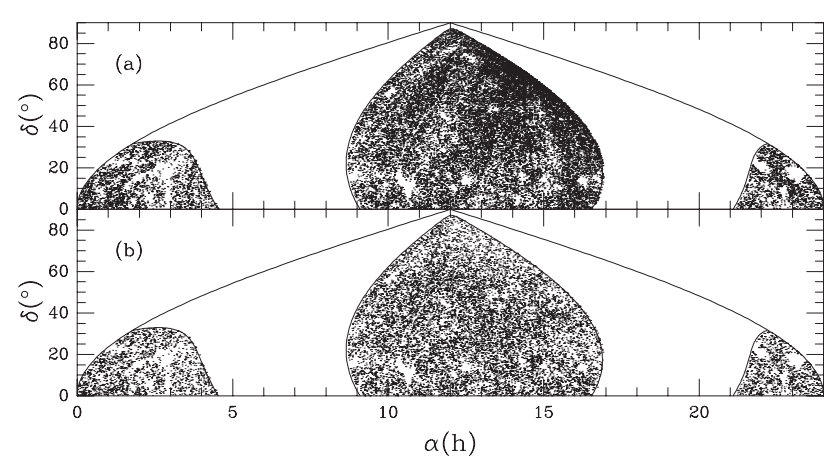

Fig. 2. Distribution over the sky of a) the 29321 ROSAT-FSC sources, b) the 15848 sources brighter than 0.02 counts $\mathrm{s}^{-1}$.

\subsection{Identification methodology}

Our aim being to identify all bright extragalactic objects associated with a ROSAT-FSC source in the area defined above, we chose to make use of the Hamburg slitless survey of the northern sky which, in principle, allows us to determine the nature of every object brighter than $O \sim 17.0$. However this would require us to visually inspect all objects within a given radius around each of the $15848 \mathrm{X}$-ray sources in the sample. This is obviously not feasible. We therefore decided to preselect all bright objects lying near the X-ray positions in the APS database, which is a catalogue of all objects visible on the Palomar Sky Survey plates, including magnitude, colour and classification as starlike or extended. But this could not be easily done as the APS was not directly available (when we started this work). The USNO catalogue is similar to the APS catalogue except that it does not classify the listed objects as starlike or extended; but it is available on CD-ROMs. It was therefore easy to select all bright USNO objects near the X-ray positions. We then sent the list of 3776 selected objects to the University of Minnesota where a batch job was run to find their APS magnitude, colour and classification.

However, as very bright stars $(O<12.0)$ are saturated on the DSS1 images and bright extended galaxies are poorly recognized by the automatic extraction procedures of both the USNO and APS databases, to find these objects we had to cross-correlate the X-ray catalogue with various catalogues of bright stars and galaxies. We ended up with 3212 objects found in the APS database, 685 bright stars and 91 additional galaxies.

The APS classification as "star-like" or "extended" were then evaluated by visual screening of the DSS2 images. 2719 APS objects were found to be "star-like" and 493 "extended". All 3212 APS objects were subsequently classified using the digitized objective prism spectra of the Hamburg Quasar Survey.

Follow-up observations were started for the "extended" objects and additional galaxies having $O_{\mathrm{APS}}<17.0$, showing the sample to be a mixture of galaxies containing $20 \%$ AGN.

The "star-like" objects were separated into several subsamples according to their APS colour and magnitude. A sample of 144 high-probability AGN candidates was selected. Based on literature data and our own follow-up spectroscopy, 83 of them are now confirmed AGN. 


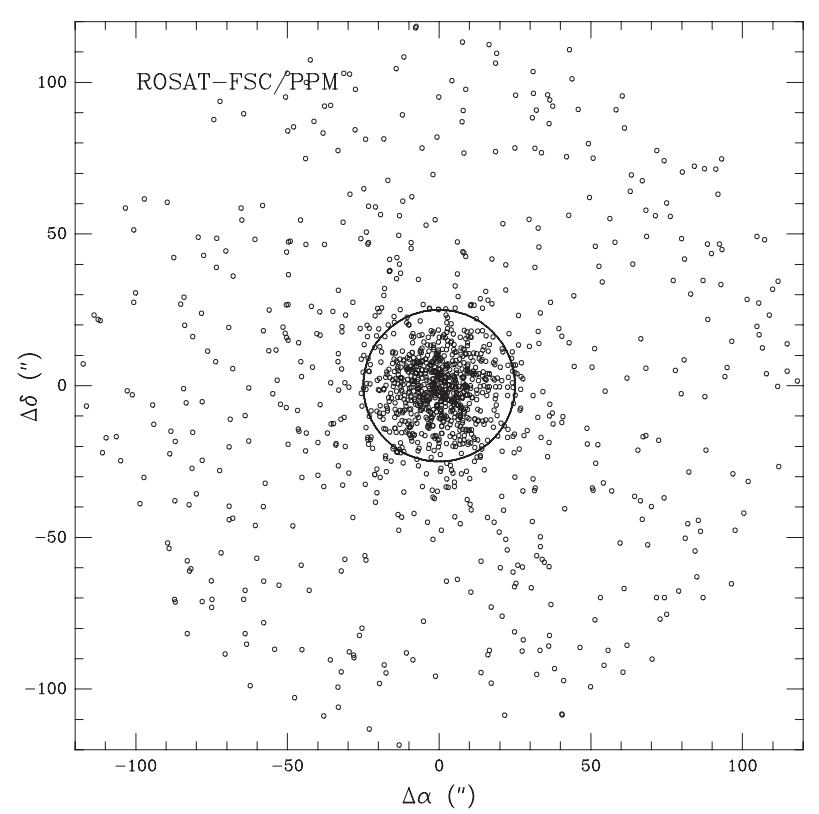

Fig. 3. Position differences for the coincidences between ROSAT-FSC $\mathrm{X}$-ray sources and PPM stars within two arcminutes. The radius of the circle is $25^{\prime \prime}$

\subsection{The USNO and APS databases}

To identify the X-ray sources with relatively bright optical counterparts we have cross-correlated our ROSAT sample with the USNO-A2.0 catalogue (Monet et al. 1996) from which we have extracted all objects in the magnitude range $O=12.0-16.5$ (4420441 i.e. $428 \mathrm{deg}^{-2}$ ). We have found 25549 objects located within $2^{\prime}$ of one of the 15848 X-ray sources.

As the USNO catalogue positions are rather poor for very bright objects, we have extracted the 1306 PPM (Positions and Proper Motions) stars (Röser \& Bastian 1991) located within $120^{\prime \prime}$ of a ROSAT position. The PPM star catalogue contains 181731 stars north of declination -2.5 , brighter than about $m_{\mathrm{pg}}=11.0$. Figure 3 shows the distribution of the position differences between the ROSAT and PPM positions. The circle drawn on the figure has a radius of $25^{\prime \prime}$. The number of coincidences within this circle is 704 (with $19 \mathrm{X}$-ray sources being associated with two PPM stars), while the expected number of chance coincidences is 27 . There are therefore 685 X-ray sources within $25^{\prime \prime}$ of at least one PPM star. We consider these 685 sources as identified; we ignored them in the following and we are left with $15163 \mathrm{X}$-ray sources.

The histogram of the separation between the optical and X-ray positions (Fig. 4) shows that, for separations larger than $35^{\prime \prime}$, there is an overwhelming majority of chance coincidences. For smaller separations, the fraction of real associations rapidly increases. This empirically defined limit is a compromise to maximize the number of real associations and minimize the number of chance coincidences. It is not straightforward to determine the fraction of real associations having a separation larger than $35^{\prime \prime}$ and therefore lost by using this limit. For separations smaller than $35^{\prime \prime}$, there are 3776 coincidences, half being expected by chance and half real. Including the PPM

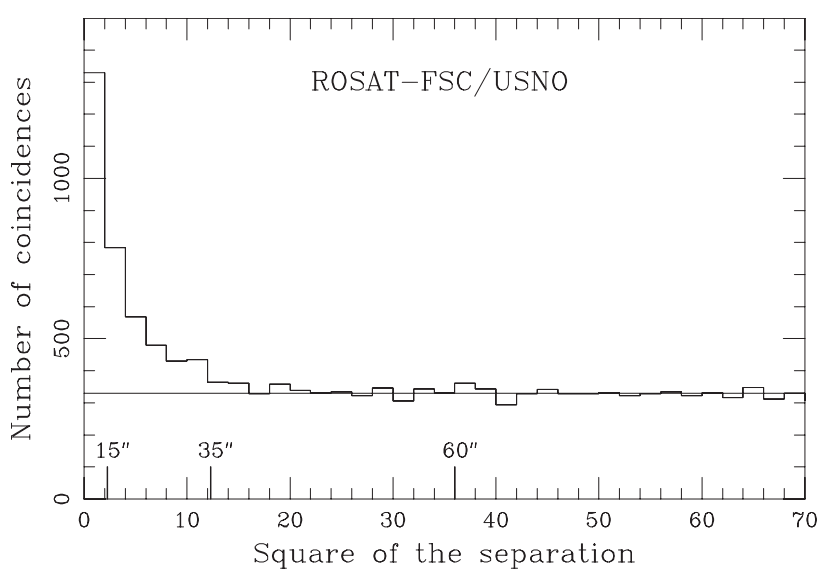

Fig. 4. Histogram of the square of the separation between the X-ray and USNO positions for the 25549 associations in units of 100 square arcsec.

stars, about $17 \%$ of the X-ray FSC sources are therefore physically associated with a relatively bright object.

Zickgraf et al. (2003) found that the $90 \%$ error radius for the X-ray positions in the BSC is $21^{\prime \prime}$. This is significantly smaller than the value of $35^{\prime \prime}$ we used for the separation limit for real associations. This difference can probably be ascribed to the differences in count rates between the two samples.

In total there are $3364 \mathrm{X}$-ray sources with at least one USNO object within 35": 2985 with a single USNO object, 348 with two, 29 with three and 2 with four.

In the APS database (Cabanela et al. 2003), the objects are classified as starlike or resolved (galaxies). The photometric calibration for the starlike objects uses a magnitude-diameter relation derived from photoelectric calibrating sequences. The $O$ magnitudes have a mean rms of $0.15-0.20$ mag over the range 14-20 mag. These magnitudes are not as reliable for objects brighter than 12th mag because of the diffraction pattern. For objects brighter than 8th mag, photometry is not available. For galaxies, the integrated magnitudes are obtained from a density-to-intensity conversion. APS derived galaxy magnitudes show no systematic photometric errors and a typical rms scatter of 0.2 to 0.3 magnitudes. The $O_{\mathrm{APS}}$ and $B$ magnitudes for stars are equal on average with a dispersion of $0.26 \mathrm{mag}$ (Mickaelian et al. 1999).

In contrast, the USNO catalogue makes no distinction between starlike and extended objects. As a consequence, the magnitudes derived for galaxies are unreliable. The typical photometric error for starlike objects is about $0.31 \mathrm{mag}$ rms (Mickaelian et al. 2001). Bright objects tend to saturate. The magnitudes reported for such objects are generally too high.

Figure 5 shows the comparison of the USNO and APS $O$ magnitudes for starlike objects (left panel) and for galaxies (right panel). While the agreement between the two sets of magnitudes is reasonably good for starlike objects, it is very poor for galaxies.

Figure 6 shows the comparison of the USNO and APS $O$ magnitudes with the NPM1 $O$ magnitudes (Klemola et al. 1987) for 95 galaxies and 81 stars. The least square 


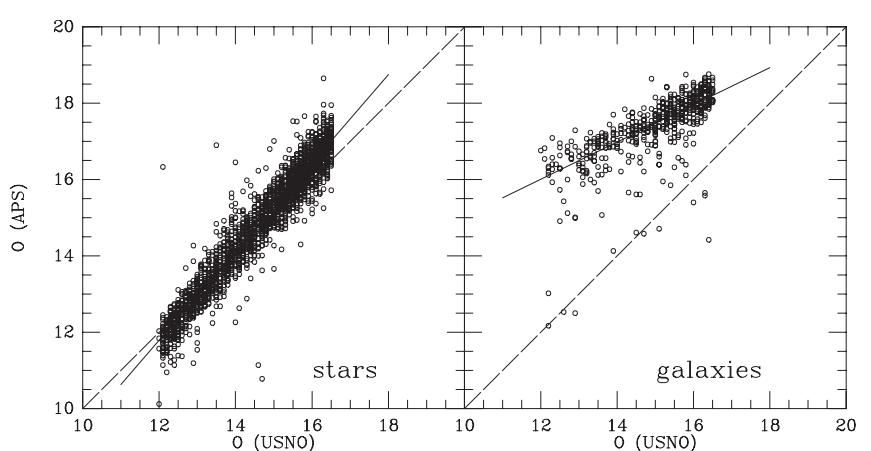

Fig. 5. Comparison of the USNO and APS $O$ magnitudes for starlike objects (left panel) and for galaxies (right panel). The solid lines represent the best linear fits between the two sets of data; the dotted lines have a slope of unity.

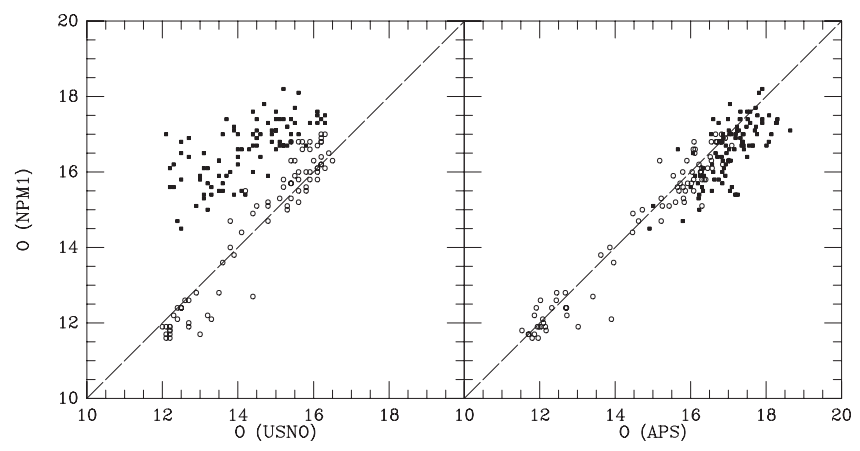

Fig. 6. Comparison of the USNO and APS $O$ magnitudes with the NPM1 $O$ magnitudes for 95 galaxies (filled squares) and 81 stars (open circles). The dotted lines have a slope of unity.

solutions for galaxies are:

$O_{\mathrm{NPM} 1 \mathrm{G}}=0.47 \times O_{\mathrm{USNO}}+11.3(\sigma=0.54 \mathrm{mag})$

$O_{\mathrm{NPM} 1 \mathrm{G}}=0.90 \times O_{\mathrm{APS}}-0.29(\sigma=0.50 \mathrm{mag})$.

This confirms that, for galaxies, the APS magnitudes are more reliable than the USNO magnitudes. We therefore extracted from the APS database objects within 10" from the 3776 USNO objects, brighter than $O=19.0$. A number of these have no APS counterpart because the required plates were not available and 49 because there was no match. We checked these 49 USNO objects on the DSS2 images (Lasker et al. 1996) and found that all of them, except one, have a starlike appearance. There are 3212 coincidences between USNO and APS objects (the 3212 sample), 2652 with starlike and 560 with extended objects.

As the APS classification based on DSS1 is not always correct, all 3212 images have been checked on the DSS2 plates. Of these, 2719 were classifd as starlike and 493 as extended. Out of the 560 APS galaxies, 135 have been reclassified as starlike; 45 of them appear as binaries so that in the APS they appear as extended. On the other hand, 68 APS starlike objects have an extended image on the DSS2.

\subsection{The Hamburg database}

All 3212 objects have been tentatively classified using the Hamburg slitless plates on the basis of a classification scheme introduced during the ROSAT-BSC identification program
Table 1. Statistics of the HQS type of 3212 optical associations with 2982 ROSAT FSC sources.

\begin{tabular}{lrr}
\hline \hline HQS types & Number & $\%$ \\
\hline AGN & 6 & 0.2 \\
Blue galaxy & 215 & 6.7 \\
Galaxy & 268 & 8.3 \\
QSO & 22 & 0.7 \\
EBL-WK & 84 & 2.6 \\
BLUE-WK & 212 & 6.6 \\
RED-WK & 87 & 2.8 \\
W-Dwarf & 20 & 0.6 \\
Star-BA & 137 & 4.3 \\
Star-FG & 1325 & 41.3 \\
Star-K & 674 & 21.0 \\
Star-M & 25 & 0.8 \\
Saturated & 81 & 2.5 \\
Overlap & 55 & 1.7 \\
Unidentified & 1 & 0.0 \\
\hline Total & 3212 & 100.0 \\
\hline
\end{tabular}

(Bade et al. 1998). The statistics of this classification is given in Table 1. Of the 493 objects classified as extended, based on direct images of the DSS2 plates, four could not be classified spectroscopically because of overlapping spectra. Based on their continua the 489 remaining objects were classified as "galaxy" (red continuum), "blue galaxy" (blue continuum) or "AGN" (prominent UV excess). Sixty one of the galaxies are member of a pair (23) or of a group (38). Among the starlike objects, 2181 were classified as stars due to absorption features in their spectra. The bright saturated objects $\left(O_{\mathrm{APS}}<12.0\right)$ were also considered as stars. Moreover 383 starlike objects, too weak for the study of absorption features, were classified on the basis of their continuum: red (RED-WK), blue (BLUEWK) and extremely blue (EBL-WK). These objects have typically $O_{\text {APS }}>16.0$. Twenty two starlike objects were classified as QSOs due to the presence of a prominent UV excess and/or of emission lines. Fifty five objects could not be classified because of overlapping spectra and one more because its spectrum did not fit the classification scheme.

The HQS classifications have been checked with already known objects. In our sample there are 107 confirmed QSOs. Two could not be classified with the Hamburg material because of overlapping spectra. Seventeen have been classified as QSOs, one as an AGN, 56 as EBL-WK, 22 as BLUEWK, two as RED-WK (J15563+0309, a low luminosity QSO with $M_{B}=-23.2$ and KUV $16355+4146$ at $z=0.765$, with $(O-E)=1.60)$ and seven as blue galaxies. These blue galaxies are in fact low luminosity QSOs $\left(M_{B}>-24.0\right)$. On the other hand eleven EBL-WK objects have been shown to be stars, as well as 21 BLUE-WK and two RED-WK objects. Clearly the EBL-WK objects are good QSO candidates as well as the BLUE-WK, although to a lesser degree. When they are not QSOs, they usually turn out to be WDs or CVs.

\section{Galaxies}

To find Seyfert galaxies we turned our attention to the 493 objects extended on the DSS2, found within $35^{\prime \prime}$ of an FSC 


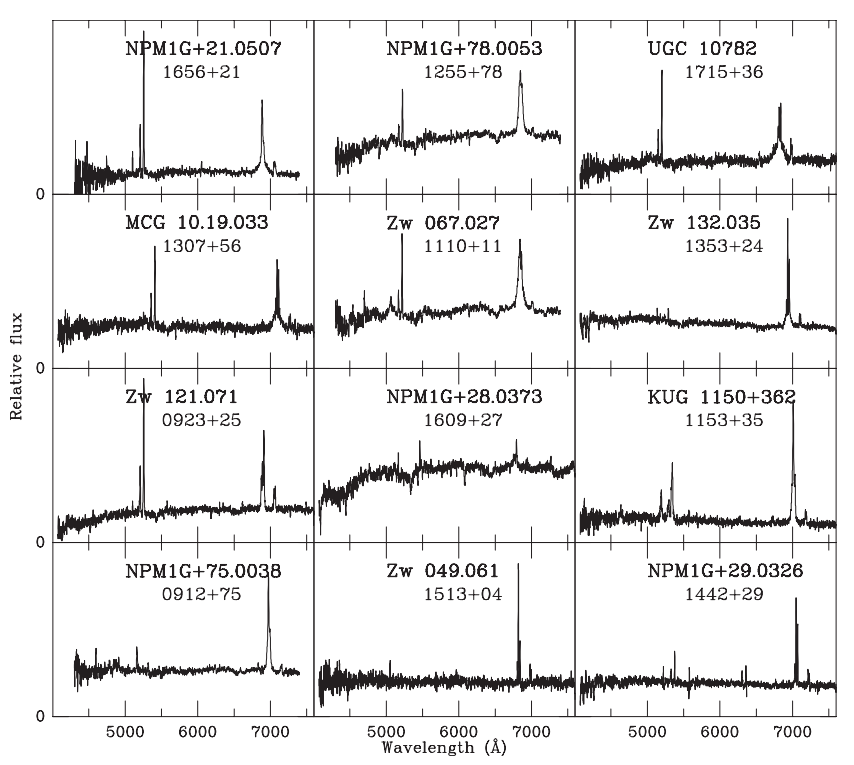

Fig. 7. Spectra of the six Seyfert 1, the three Seyfert 2 and three of the five starburst galaxies confirmed by our spectroscopic observations at $\mathrm{BAO}$ and OHP.

source. If there were no real association, the number of coincidences within $15^{\prime \prime}$ would be 0.184 times the number of coincidences within $35^{\prime \prime}$ (the ratio of the respective areas). On the other hand, if all associations were real, Fig. 4 shows that almost all identifications would be located within $35^{\prime \prime}$ and about $50 \%$ within $15^{\prime \prime}$. In a sample in which the fraction of coincidences within $15^{\prime \prime}$ is $x$, the fraction $y$ of true associations would be $y=(x-0.184) /(0.5-0.184) .218(x=44)$ of the 493 galaxies are within $15^{\prime \prime}$ of an X-ray source which shows that a large fraction of them $(y \sim 80 \%)$ are genuine associations.

As we have seen above, sixty eight of the objects found to be extended on the DSS2 were classified as starlike in the APS data base. Three of them (NGC 1085, IC 2439 and KUG 2323+85) were previously found to be bright galaxies and are included in Table 2 . The 65 others are probably compact galaxies; 47 are classified as "blue galaxies" on the slitless HQS spectra (in fact, ten of them are already known to be Seyfert 1s or low luminosity QSOs).

As the spectroscopic observations took place before we had the opportunity to check the APS classification on the DSS2 images (which is a rather tedious procedure as it is done visually rather than automatically), these 65 objects were not included in our original list of galaxy candidates.

Of the 493 extended objects, 155 are brighter than $O_{\mathrm{APS}}=$ 17.0. Ninety four of them were classified as galaxies in the APS database. To these 94 galaxies we added 91 bright galaxies not appearing in the USNO or APS databases but found by crosscorrelating the ROSAT FSC with catalogues of bright galaxies (NGC, UGC, NPM1G, Mark, Zw, etc.). As the surface density in these catalogues is small, we accepted as identification all coincidences within $35^{\prime \prime}$. These 185 galaxy candidates are listed in Table 2. The redshift of 130 of them were known from the literature.

We have observed 34 of the galaxies without a previously known redshift at the $\mathrm{BAO}$ and $\mathrm{OHP}$ observatories. The BAO observations were made during the period March 3 to 10, 2002 with the 2.6-m telescope using the SCORPIO spectral camera attached to the prime focus. The combination of the detector, a $2063 \times 205816 \times 16 \mu$ m pixel Loral Lick3 CCD, with a $600 \mathrm{~g} \mathrm{~mm}^{-1}$ grism resulted in the spectral range 3900$7400 \AA$ with a dispersion of $1.7 \AA$ pixel $^{-1}$. The slit width was 1". 8 (4.3 pixels) and was oriented EW. The spectral resolution was $5 \AA$ A $F H M$. The exposure time was usually $45 \mathrm{~min}$. The OHP observations were made between April 15 and 19, 2002 with the 1.93-m telescope and the spectrograph CARELEC (Lemaitre et al. 1989) attached to the Cassegrain focus. The detector was a $2048 \times 102413.5 \times 13.5 \mu \mathrm{m}$ pixel EEV CCD. The dispersion was $1.75 \AA$ pixel $^{-1}$ and the spectral range 4075$7715 \AA$. The slit width was 2 '” 0 , corresponding to 3.7 pixels, and the resolution was $5.7 \AA F W H M$. The exposure time was usually $20 \mathrm{~min}$. We found 13 absorption line galaxies, five starburst galaxies, six Seyfert 1, three Seyfert 2 and seven unclassifiable weak emission line galaxies. Figure 7 shows the spectra of the Seyfert 1 and 2 galaxies and of three starburst galaxies.

In total the redshift of 164 of the galaxies is known. We have computed their $0.1-2.4 \mathrm{keV}$ X-ray luminosity. Sixtythree are smaller than $10^{42} \mathrm{erg} \mathrm{s}^{-1}$ and forty-five, greater than $10^{42.5} \mathrm{erg} \mathrm{s}^{-1}$. As there are probably no starburst galaxies having an $0.1-2.4 \mathrm{keV} \mathrm{X}$-ray luminosity substantially higher than $10^{42} \mathrm{erg} \mathrm{s}^{-1}$ (Moran et al. 1996; Condon et al. 1998), most of the 45 high X-ray luminosity galaxies must be AGN. Among the 16 which have been classified, four are starbursts and 12 AGN. The 63 low X-ray luminosity galaxies could possibly be absorbed Seyfert 2s, low-luminosity AGN as well as starbursts, or elliptical galaxies with hot gas (Hornschemeier et al. 2001; 2003; Comastri et al. 2003; Severgnini et al. 2003). Several of our low X-ray luminosity sources have been classified as Liners, Seyfert 2s, Seyfert 1.9 or 1.8.

\section{QSOs}

Figure 8a shows the APS $O-E$ colour index vs. the $O$ magnitude for the 2652 starlike objects in the 3212 sample. Figure $8 b$ is the same for objects within $15^{\prime \prime}$ of a ROSAT-FSC source. These figures were divided into four zones.

Objects brighter than $O_{\mathrm{APS}}=13.5$ (zone I) are most probably bright stars. Indeed among the 576 objects in this zone, 84 could not be classified on the Hamburg slitless spectra because of saturation or overlap, but all others are stars (25 BA, 416 FG and $51 \mathrm{~K}$ ). Objects located above the diagonal line (zone II) are very red and are likely to be late-type stars. Fig. $8 \mathrm{c}$ shows that indeed $\mathrm{K}$ and $\mathrm{M}$ stars are mostly located in this zone. Among the 709 objects in this zone, 13 are affected by overlapping or saturation, 685 are stars (including $437 \mathrm{~K}$ stars); 11 were too weak to be classified.

We cross-correlated the FSC with the current version of the Véron-Cetty \& Véron (2001) AGN catalogue. We found 76 coincidences within $35^{\prime \prime}$ with a previously known AGN brighter than $O_{\text {USNO }}=16.5$ (they are listed in Table 3); 68 have magnitude and colour in the APS database. Figure 9 is a plot of the APS $O-E$ colour index vs. the $O$ magnitude for these $68 \mathrm{AGN}$; $87 \%(59 / 68)$ are located in zone IV defined by $O_{\mathrm{APS}}>15.5$ and $O-E<1.2$. With this limit on $O-E$, we exclude red QSOs; 


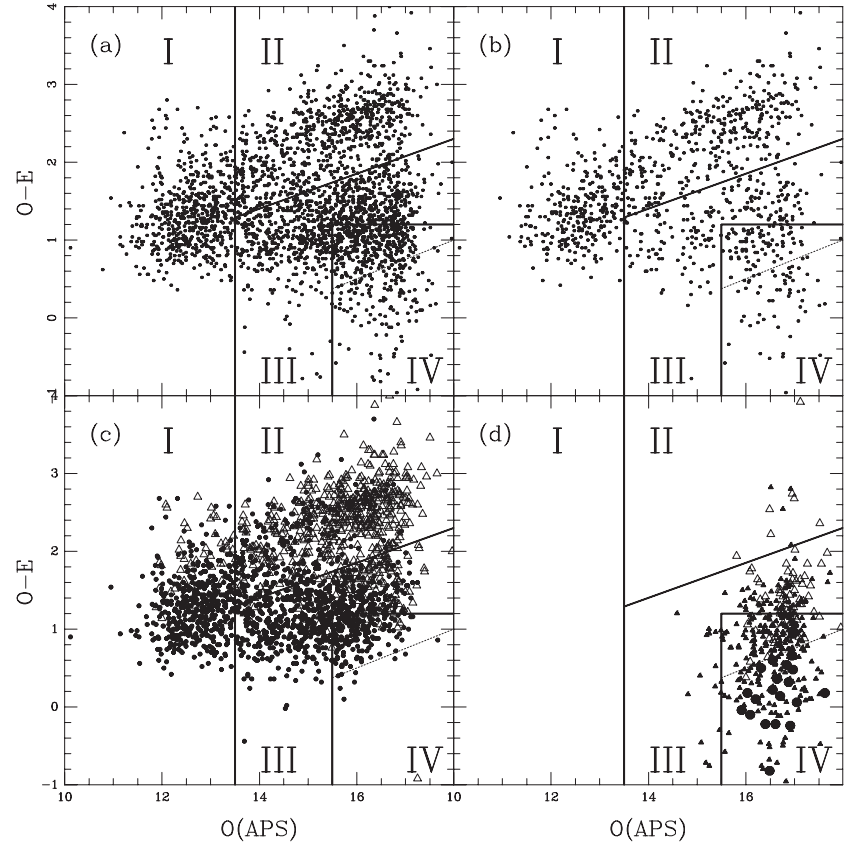

Fig. 8. a) APS $O-E$ colour indices vs. $O$ magnitudes for all starlike candidate identifications within $35^{\prime \prime}$; b) the same for coincidences within $15^{\prime \prime} ; \mathbf{c}$ ) the same for the A to G stars (filled circles) and the K and $\mathrm{M}$ stars (open triangles) as classified according to the Hamburg scheme; d) the same for the BLUE-WK and EBL-WK objects (filled triangles), the RED-WK objects (open triangles) and the QSOs (filled circles).

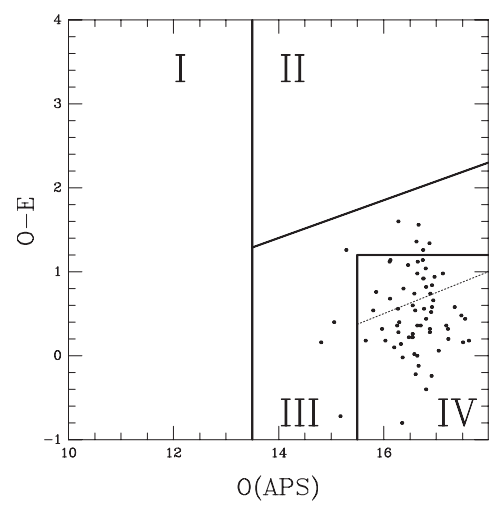

Fig. 9. APS $O-E$ colour indices vs. $O$ magnitudes for 68 confirmed QSOs.

this is unavoidable because of the very large number of stars with $O-E>1.2$. These objects can be recovered by examination of the slitless spectra. Figures $8 \mathrm{~d}$ and 9 show that the distributions of the representative points of the EBL-WK and BLUE-WK objects on the one hand and of that of QSOs on the other hand are quite similar.

Figure 10 shows the differences between the X-ray and optical positions for the starlike objects in the 3212 sample in each of the four zones defined above. In the first two zones there is an obvious concentration towards the centre showing that most of the coincidences are genuine associations. In contrast the distribution of the points in zone III is quite uniform suggesting that most of these coincidences are due to chance.

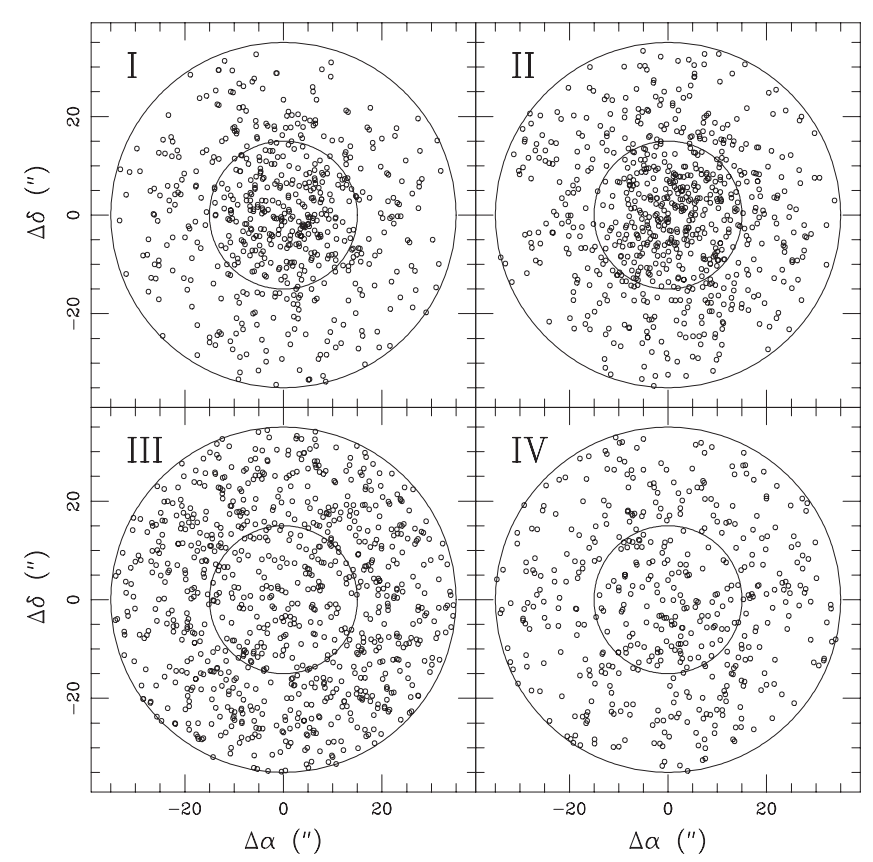

Fig. 10. Position differences between the ROSAT-FSC X-ray sources and APS starlike objects located in each of the four zones previously defined. The circles have a radius of $15^{\prime \prime}$ and $35^{\prime \prime}$, respectively.

In zone III, the observed fraction of coincidences within $15^{\prime \prime}$ is $x=0.20$, suggesting that there are indeed very few true associations $(y=0.05)$. On the other hand, in zone I and II, we found $x=0.47$ and 0.45 respectively showing that basically all objects (90 and $84 \%$ respectively) are real associations.

For the 212 starlike objects classified as BLUE-WK on the Hamburg slitless spectra, we found $x=0.24$, so the fraction of true coincidences is about $18 \%$, while for the 84 EBL-WK objects, $x=0.51$ and, within the statistical uncertainties, all are genuine associations.

Out of the 2652 starlike objects, zone IV contains 476 coincidences within $35^{\prime \prime}$ and $136(x=0.28)$ within $15^{\prime \prime}$ of an $\mathrm{X}$-ray source, implying that $y=0.32$; therefore 153 of these coincidences are likely to be genuine associations, 76 being located within $15^{\prime \prime}$. They could be blue stars (WD, CV, sd), or more probably QSOs. We have divided zone IV in two subregions (see the dotted line in Fig. 8). In the upper region (IVb), only $23 \%$ of the coincidences are within $15^{\prime \prime}$ and therefore a small fraction of the 332 coincidences are genuine. In the lower region (IVa) containing the bluest objects, $41 \%$ of the coincidences are located within $15^{\prime \prime}$ from the X-ray source, suggesting that $\sim 70 \%$ of the 144 objects located in this zone are real identifications. Among them are 41 known QSOs. The 103 other candidates, of which seven are known stars, are listed in Table 4. We have spectroscopically observed 91 of them. The observations were carried out during three observing runs (May and August 2002 and May 2003) with the 2.1-m telescope of the Guillermo Haro Astrophysical Observatory (OAGH) located in Cananea (Sonora, Mexico), operated by INAOE, and one run (September 2003) with the OHP 1.93-m telescope and the same instrument setting as for the observations of the galaxies (see Sect. 3). The 2.1-m telescope was equipped with the LFOSC focal reducer (Zickgraf et al. 1997). The slit mask and 


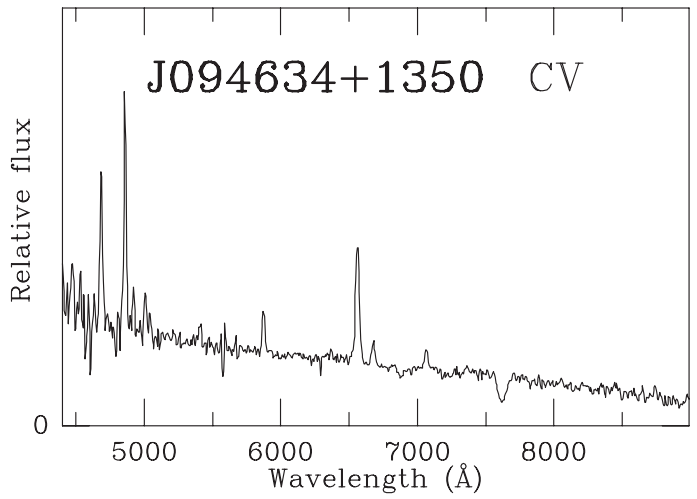

Fig. 11. Spectrum of a cataclysmic variable observed at OAGH.

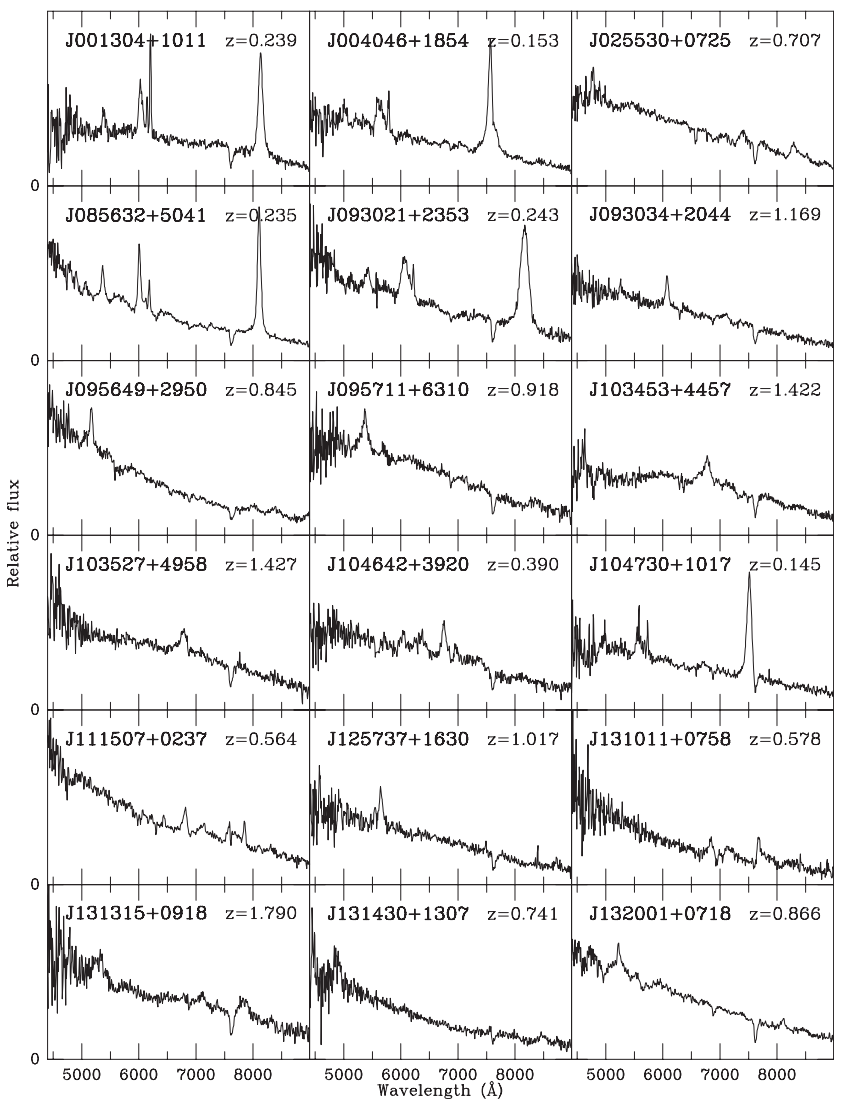

Fig. 12. Spectra of 18 QSOs from Table 4 observed at OAGH.

the lower dispersion grism were used, giving a wavelength coverage from $4200 \AA$ to $9000 \AA$ and a dispersion of $8.2 \AA$ pixel $^{-1}$. We found 49 stars (including a CV the spectrum of which is shown in Fig. 11), 38 QSOs, 1 Seyfert 1, two NLS1s, one BL Lac object (their spectra are displayed in Figs. 12-14). Five candidates have not yet been observed.

Altogether, there are, in region IVa, 83 confirmed AGN in a total of 139 spectroscopically observed starlike objects $(60 \%)$.

But only $60 \%$ (41/68) of all previously known AGN with APS colour fall in zone IVa showing the limits of the efficiency of the procedure we have adopted. The others are located in zone IVb, or not far from it, in a region where chance coincidences between stars and X-ray sources become non

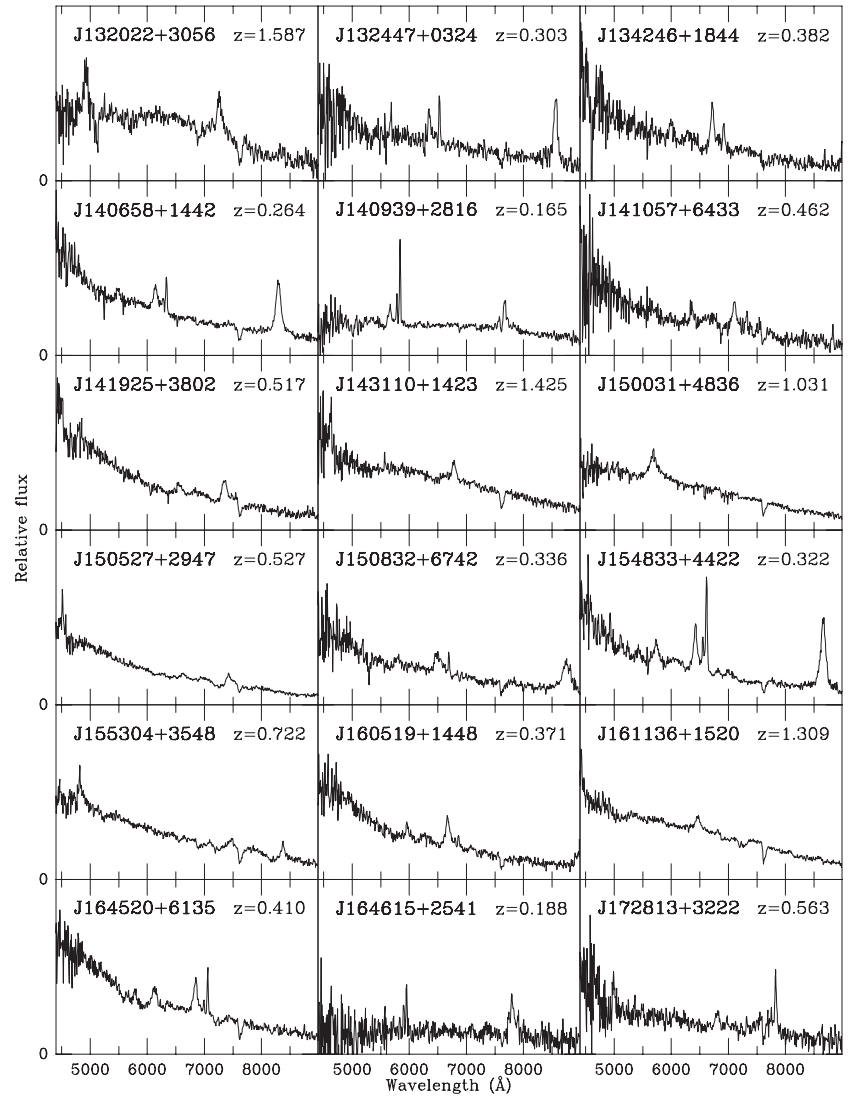

Fig. 13. Spectra of 18 QSOs from Table 4 observed at OAGH.

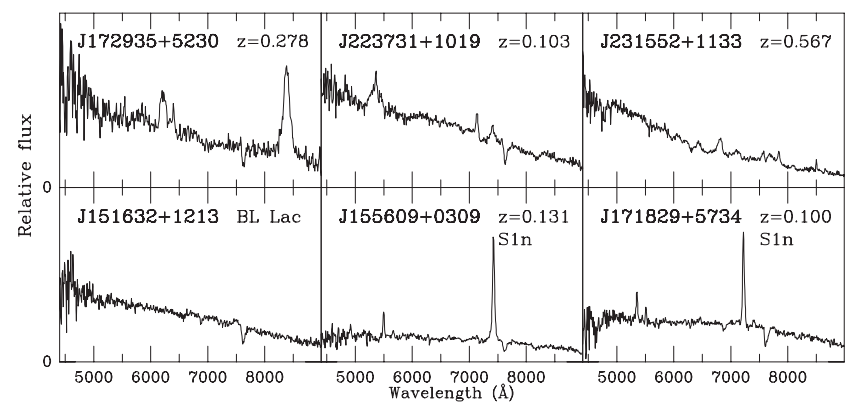

Fig. 14. Spectra of six objects from Table 4 (one Seyfert 1, one QSO, two BL Lac objects and two NLS1s) observed at OAGH.

negligible. Here the Hamburg slitless spectra may turn out to be most useful in separating AGN from stars.

\section{Discussion}

\subsection{Identification procedure}

We have seen that the galaxies found within $35^{\prime \prime}$ of a ROSAT source have a high probability of being genuine identifications $(\sim 80 \%)$ and therefore we included all of them in our spectroscopic program.

In the case of starlike objects, the situation is more complex due to the facts that a large fraction of the X-ray sources are in fact X-ray stars and that, the surface density of stars being relatively large, there is a non negligible number of chance coincidences. The use of the $O-E$ vs. $O$ diagram turns out to 
Table 5. Number and percentage of radio quiet and radio loud X-ray detected QSOs in the Véron-Cetty \& Véron catalogue. Columns 1 and 2: $O_{\mathrm{USNO}}$ range, Col. 3: number of QSOs, Col. 4: number of sources brighter than 0.02 count $\mathrm{s}^{-1}$ in the ROSAT catalogues, Col. 5: percentage of ROSAT detected QSOs; Col. 6 to 8: the same for the radio loud QSOs.

\begin{tabular}{rr|rrr|rrr}
\hline \hline & \multicolumn{4}{c}{ Radio quiet } & \multicolumn{3}{c}{ Radio loud } \\
\hline$O_{\min }$ & $O_{\max }$ & $n$ & $n_{\mathrm{X}}$ & $\%$ & $n$ & $n_{\mathrm{X}}$ & $\%$ \\
\hline & $<15.5$ & 80 & 58 & 72 & 9 & 7 & 78 \\
15.5 & 16.0 & 90 & 48 & 53 & 21 & 17 & 81 \\
16.0 & 16.5 & 243 & 101 & 42 & 49 & 37 & 76 \\
16.5 & 17.0 & 433 & 144 & 33 & 99 & 60 & 61 \\
17.0 & 17.5 & 679 & 101 & 15 & 172 & 77 & 45 \\
17.5 & 18.0 & 900 & 60 & 7 & 201 & 59 & 29 \\
18.0 & 18.5 & 977 & 23 & 2 & 169 & 41 & 24 \\
18.5 & 19.0 & 1040 & 13 & 1 & 107 & 11 & 10 \\
19.0 & 19.5 & 475 & 4 & 1 & 41 & 5 & 12 \\
19.5 & 20.0 & 87 & 0 & 0 & 18 & 5 & 28 \\
\hline
\end{tabular}

be quite efficient to eliminate bright stars and late type stars. However, although in our region IVa most objects are genuine identifications, in zones IVb and III there are many chance coincidences with main sequence stars. The use of the Hamburg slitless spectra in these zones is crucial to separate QSOs from stars. In addition, Seyfert 1 galaxies are often compact and classified as starlike in the APS. Moreover they usually have a relatively red $O-E$ colour; as a consequence they easily escape detection by our automatic procedure and classification on the basis of the slitless spectra is necessary.

The relatively poor positional accuracy of the ROSAT survey $\left(\sigma \sim 15^{\prime \prime}\right)$ makes it difficult to identify objects weaker than $\sim 16.5$; but this limiting magnitude is well fitted by the magnitude limit below which no recognition of absorption features is possible in the Hamburg slitless spectra.

\subsection{Completeness of our QSO sample}

We have seen that only $85 \%$ of the bright USNO objects have a counterpart in the APS due mainly to the incompleteness of the data base. Moreover only $60 \%$ of the known QSOs fall into our zone IVa to which we have restricted our search. Therefore our sample is expected to be no more than $50 \%$ complete.

On the other hand, as our aim is to find all AGN brighter than a given optical magnitude, we have to answer the following question: how many such AGN are missed if we identify all bright AGN in a flux limited X-ray survey?

The current version of the Véron \& Véron QSO catalogue contains 5893 QSOs at $\delta>0^{\circ},|b|>30^{\circ}$, with $B<20.0$ and $z>0.10$, found in the course of radio or optical surveys (QSOs found as the identification of a X-ray source have been ignored). Of these, 871 are located within $35^{\prime \prime}$ from a ROSATBSC or -FSC source (stronger than 0.02 count $\mathrm{s}^{-1}$ ). We consider them as genuine identifications; 319 are radio loud and 552 radio quiet.

On average, QSOs with higher optical luminosity have higher X-ray luminosity. According to Maccacaro et al. (1988), $\log \left(F_{\mathrm{X}} / F_{V}\right)=\log \left(F_{\mathrm{X}}\right)+0.4 \times V+5.37$ where $F_{\mathrm{X}}$ is the

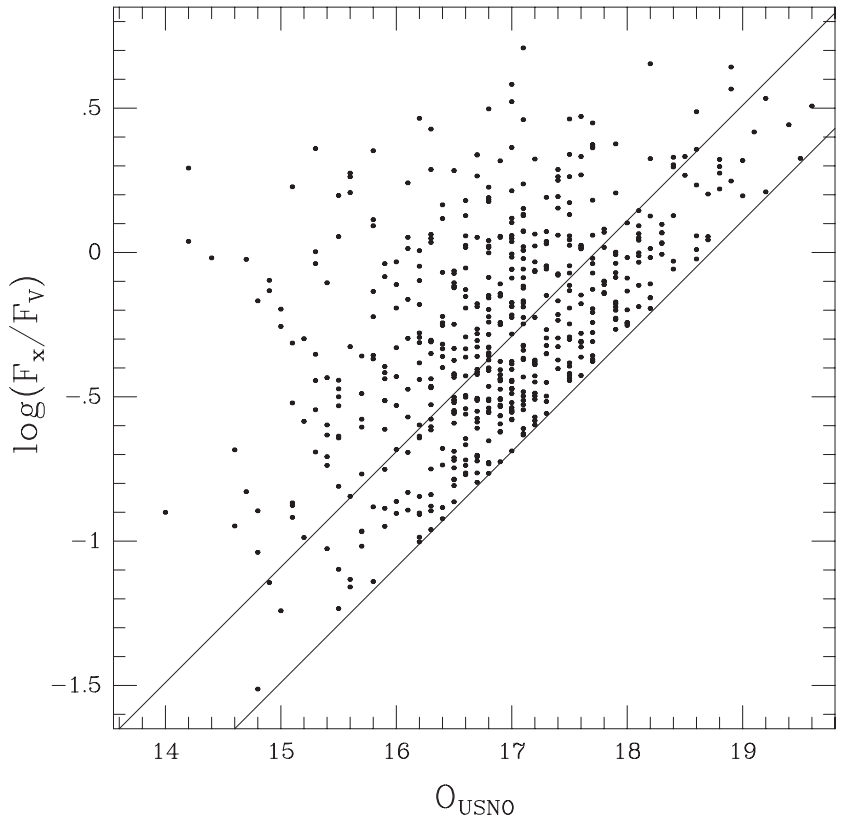

Fig. 15. Plot of $\log \left(F_{\mathrm{X}} / F_{V}\right)$ vs. $O_{\mathrm{USNO}}$ for 552 radio quiet QSOs. The two diagonal lines correspond to the sensitivity limits of the BSC and FSC ROSAT survey (0.05 and 0.02 count s $^{-1}$, respectively).

X-ray flux in the $0.3-3.5 \mathrm{keV}$ band in $\mathrm{erg} \mathrm{cm}^{-2} \mathrm{~s}^{-1}$ and $F_{V}=$ $-0.4 \times V-8.42$ is the flux in the $V$ band in the same units. For the ROSAT survey, this translates into: $\log \left(F_{\mathrm{X}} / F_{V}\right)=\log$ (PSPC counts $\left.\mathrm{s}^{-1} \times 10^{-11}\right)+0.4 \times V+5.37$ where $F_{\mathrm{X}}$ is the $\mathrm{X}$-ray flux in the $0.1-2.4 \mathrm{keV}$ band, assuming a conversion factor of 1 PSPC count s $\mathrm{s}^{-1}$ for a flux of $10^{-11} \mathrm{erg} \mathrm{cm}^{-2} \mathrm{~s}^{-1}$ in this band which corresponds to an unabsorbed source with a photon in$\operatorname{dex} \Gamma=2.3$, a typical value for QSOs (Voges et al. 1999).

Figure 15 shows $\log \left(F_{\mathrm{X}} / F_{V}\right)$ vs. $O_{\mathrm{USNO}}$ for the 552 radio quiet QSOs. Table 5 gives the fraction of ROSAT detected QSOs vs. their optical magnitude for both the radio quiet and the radio loud samples.

At $O_{\mathrm{USNO}}=16.0$, about half of the optically selected QSOs are not detected in X-ray (Table 5) and therefore have $\log \left(F_{X} / F_{V}\right)<-1$. Consequently the median value of their $\mathrm{X}$-ray to optical flux ratio is $\sim-1.0$. Figure 15 suggests that $\sigma \sim 0.5$.

Zickgraf et al. (2003) found that the median value of $\log \left(F_{\mathrm{X}} / F_{V}\right)$ for $\mathrm{X}$-ray selected QSOs is 0.39 . The fact that these objects are significantly stronger X-ray sources than optically selected QSOs is due to the much higher frequency of the survey. Indeed Williams \& Bridle (1967) and Kellermann et al. (1968) have shown that the relative numbers of sources with given spectral indices depend on the frequency at which the sample is selected. A sample selected at a low frequency (optical) will include a greater proportion of sources with high values of the spectral index than will a similar sample selected at a high frequency (X-ray).

In the simple case where the distribution of the spectral indices $\alpha$ in a complete sample of sources selected at a wavelength $\lambda_{\mathrm{o}}$ is Gaussian with a mean spectral index $\alpha_{\mathrm{o}}$ and a standard deviation $\sigma_{\alpha}$, the distribution of the spectral indices of sources selected at another wavelength $\lambda_{\mathrm{X}}$ will also be 
Gaussian with the same standard deviation $\sigma_{\alpha}$, but with a mean spectral index $\alpha_{\mathrm{X}}$ given by: $\alpha_{\mathrm{X}}=\alpha_{\mathrm{o}}+\mu \times \sigma_{\alpha}^{2} \times \ln \left(\lambda_{\mathrm{X}} / \lambda_{\mathrm{o}}\right)$. In this expression $\mu$ is the exponent in the population law $N(S) \sim S^{-\mu}$ giving the number of sources with flux densities in excess of a specified value $S$ (Williams \& Bridle 1967). With $\mu=1.87, \lambda_{\mathrm{o}}=5000 \AA$ and $\lambda_{\mathrm{X}}=12.4 \AA(1 \mathrm{keV})$, we find that to get the observed increased value of $F_{\mathrm{X}} / F_{V}$ by a factor 25 , corresponding to $\alpha_{\mathrm{X}}-\alpha_{\mathrm{o}}=0.54$, a value of $\sigma_{\alpha}=0.22$ is required, i.e. $\sigma=0.57$, where $\sigma=2.60 \times \sigma_{\alpha}$ is the standard deviation of $\log \left(F_{\mathrm{X}} / F_{V}\right)$. This is in reasonable agreement with our estimated value of $\sigma \sim 0.5$.

Table 5 confirms that radio loud QSOs are brighter X-ray sources than radio quiet QSOs. In addition we found that, for BL Lac objects, the mean $F_{\mathrm{X}} / F_{V}$ ratio is larger than that of the radio loud QSOs as previously shown by Stocke et al. (1985). BL Lac brighter than 15.5 are all detected by ROSAT while at $O_{\text {APS }}=20.0,50 \%$ are still detected.

\subsection{The surface density of bright QSOs}

The surface density of bright QSOs is difficult to determine because these objects are rare and therefore large areas of the sky have to be surveyed to find a substantial number. The Bright QSO Survey (BQS) of Schmidt \& Green (1983) covering $10714 \mathrm{deg}^{2}$, contains 69 QSOs brighter than $M_{B}=-24.0$ and $B=16.16$, corresponding to $0.0064 \pm 0.0008 \mathrm{deg}^{-2}$. A number of works suggest that this value is substantially underestimated and that the completeness of the BQS is in the range 30\%-70\% (Wampler \& Ponz 1985; Goldschmidt et al. 1992; La Franca \& Cristiani 1997; Wisotzki et al. 2000; Mickaelian et al. 2001).

Grazian et al. (2002) have undertaken a program similar to ours. They cross-correlated the ROSAT-BSC with the Digitized Sky Survey in the southern hemisphere to find all coincidences between X-ray sources and stellar objects brighter than $B_{\mathrm{J}}<15.13$. After spectroscopic observations of the candidates, they obtained a sample of $111 \mathrm{AGN}$ in an area of $5660 \mathrm{deg}^{2}, 57$ being brighter than $M_{B}=-24.0$. But the distribution of absolute magnitudes is rather odd, 46 being greater than -25.0 and 5 smaller than -26.0 . We checked the appearance of some of the low redshift objects on the DSS images; they are clearly galaxies; their $O$ magnitude in the APS data base is much greater than the $B$ magnitude given by Grazian et al. and the absolute magnitudes computed with these $O$ magnitudes are definitely greater than -24.0 . The true number of QSOs in this sample is therefore unknown, but certainly significantly smaller than 57 .

There are, in our region of interest $\left(10313 \mathrm{deg}^{2}\right), 503$ known QSOs $\left(M_{B}<-24.0\right.$ i.e. $\left.M_{O}<-24.4\right)$ brighter than $O_{\mathrm{USNO}}=16.5$, i.e. $B=16.9$ (on the average, the $O_{\mathrm{USNO}}$ magnitudes are brighter than the photoelectric $B$ magnitudes: $<O_{\mathrm{USNO}}-B>=-0.38$; Mickaelian et al. 2001). 191 are associated with a BSC source, 84 with a FSC source and 228 are not detected by ROSAT. Here we use USNO rather than APS $O$ magnitudes because the selection has been made with the USNO data base; using the APS magnitudes, our sample would not be complete to $O=16.5$.

These 503 QSOs correspond to a surface density of $0.041 \pm$ 0.002 QSO $\mathrm{deg}^{-2}$ brighter than $B=16.9$. If the integrated number-magnitude relation has a slope equal to $b=\mu / 2.5=$ 0.75 (Sandage \& Luyten 1969), the surface density of QSOs brighter than $B=16.16$ is $0.0114 \mathrm{deg}^{-2}$. Of these QSOs 142 are brighter than $O_{\mathrm{USNO}}=15.8(B=16.2)$, corresponding to $0.014 \pm 0.001 \mathrm{deg}^{-2}$, in agreement with the preceding value, confirming that the value of $b$ used is reasonable.

The QSO counts are systematically affected by the photometric errors on the magnitudes as these errors scatter many more objects toward bright magnitudes than it does toward faint magnitudes. Assuming that the error distribution is Gaussian, with a dispersion $\sigma$, the correction to be applied to the observed counts is a factor $10^{(b+1) \sigma^{2} / 2}$ (Eddington 1940). With $b=0.75$ and $\sigma=0.31$, the true QSO surface density is smaller by 1.21 than the observed one or, at $B=16.16,0.011 \pm 0.002 \mathrm{deg}^{-2}$. This is already $78 \%$ larger than the original Schmidt \& Green estimate.

Sixty seven of the known QSOs $\left(M_{O}<-24.4\right)$ with available APS magnitudes are associated with a ROSAT-FSC source, 35 being located in zone IVa (Table 3 ). In this zone, we have found 33 additional such objects (see Table 4), almost doubling the number of known QSOs at this limiting magnitude.

We should note in addition that, among the 84 EBL-WK objects, 14 have not yet been spectroscopically observed, while 5 of the QSOs candidates in Table 4 have still to be observed. This may increases the number of new bright QSOs.

\section{Conclusion}

We have undertaken a program of systematic identification of optically bright AGN in both the BSC- and FSC-ROSAT catalogues. In this paper we present preliminary results concerning the FSC catalogue. We describe the method used which consists of first cross-correlating the USNO and APS databases with the X-ray catalogue, then checking the nature of the candidates by looking at the slitless Hamburg spectra.

Among the starlike objects brighter than $B=16.9$ we have recovered 35 previously known QSOs in the region of the $O /(O-E)$ diagram containing $60 \%$ of the bluest QSOs and discovered 33 new ones confirming that, even at these relatively bright magnitudes, available surveys are significantly incomplete.

The total number of previously known QSOs brighter than $B=16.2$ corresponds to a surface density $78 \%$ higher than the original Schmidt \& Green value. But we have shown that, in the subsample studied here i.e. the QSOs associated with a ROSAT-FSC source, the completeness is at best 50\%. Although we cannot generalize this to the whole sample, it is not unlikely that the true surface density is significantly higher than the number derived here.

We found that at this magnitude and at a flux limit of 0.02 counts $^{-1}$, only $40 \%$ of all QSOs are detected by ROSAT. 
Acknowledgements. R.M. and V.Ch. acknowledge CONACyT research grants J32178-E and 39560-F. This work is partly based on observations obtained with 2.6-m telescope at the Byurakan Astrophysical Observatory, Armenia, the 1.93-m telescope at the Observatoire de Haute Provence (CNRS), France and the 2.1-m at the Guillermo Haro Astrophysical Observatory, Cananea, Mexico. The authors acknowledge the hospitality of INAOE during the Guillermo Haro Workshop 2003, at which time the scientific conclusions presented here were discussed. They are grateful to Dr. J. Cabanela for his help with the use of the APS databases which are supported by the National Science Foundation, the National Aeronautics and Space Administration, and the University of Minnesota, and are available at http://aps.umn.edu/. The Digitized Sky Survey was produced at the Space Telescope Science Institute (STScI) under U.S. Government grant NAG W-2166.

\section{References}

Bade, N., Engels D., Voges W., et al. 1998, A\&AS, 127, 145

Cabanela, J. E., Humphreys, R. M., Aldering, G., et al. 2003, PASP, 115,837

Comastri, A., Brusa, M., Mignoli, M., \& Hellas team 2003, AN, 324, 28

Condon, J. J., Yin, Q. F., Thuan, T. X., \& Boller, T. 1998, AJ, 116, 2682

Eddington, A. S. 1940, MNRAS, 100, 354

Goldschmidt, P., Miller L., La Franca, F., et al. 1992, MNRAS, 256, $65 \mathrm{P}$

Grazian, A., Omizzolo, A., Corbally, C., et al. 2002, AJ, 124, 2955

Hagen, H.-J., Groote, D., Engels, D. \& Reimers, D. 1995, A\&AS, 111, 195

Hornschemeier, A. E., Brandt, W. N., Garmire, G. P., et al. 2001, ApJ, 554,742

Hornschemeier, A. E., Bauer, F. E., Alexander, D. M., et al. 2003, AJ, 126,575

Kellermann, K. I., Pauliny-Toth, I. I. K., \& Davis, M. M. 1968, Astrophys. Lett., 2, 105
Klemola, A. R., Jones, B. F., \& Hanson, R. B. 1987, AJ, 94, 501

La Franca, F., \& Cristiani, S. 1997, AJ, 113, 1517

Laor, A., Fiore, F., Elvis, M., Wilkes, B. J., \& McDonnel, J. C. 1997, ApJ, 477, 93

Lasker, B. M., Doggett, J., McLean, B., et al. 1996, ASP Conf. Ser., 101,88

Lemaître, G., Kohler, D., Lacroix, D., Meunier, J.-P., \& Vin, A. 1989, A\&A, 228, 546

Maccacaro, T., Gioia, I. M., Wolter, A., Zamorani, G., \& Stocke, J. T. 1988, ApJ, 326, 680

Mickaelian, A. M., Gonçalves, A. C., Véron-Cetty, M.-P., \& Véron, P. 1999, Astrophysics, 42, 5

Mickaelian, A. M., Gonçalves, A. C., Véron-Cetty, M.-P., \& Véron, P. 2001, Astrophysics, 44, 21

Monet, D., Bird, A., Canzian, B., et al. 1996, USNO-A2.0, US Naval Observatory, Washington DC

Moran, E. C., Halpern, J. P., \& Helfand, D. J. 1996, ApJS, 106, 341

Odewahn, S. C., Stockwell, E. B., Pennington, R.L., \& Humphreys, R. M., 1992, AJ, 103, 318

Röser, S., \& Bastian, U. 1991, PPM star catalogue, Astronomisches Rechen-Institut Heidelberg

Sandage, A., \& Luyten, W. J. 1969, ApJ, 155, 913

Schmidt, M. \& Green, R. F. 1983, ApJ, 269, 352

Schwope, A. D., Hasinger, G., Lehmann, I., et al. 2000, AN, 321, 1

Severgnini, P., Caccianiga, A., Braito, V., et al. 2003, A\&A, 406, 483

Stocke, J. T., Liebert, J., Schmidt, G., et al. 1985, ApJ 298, 619

Tananbaum, H., Avni, Y., Branduardi, G., et al. 1979, ApJ, 234, L9

Véron-Cetty, M.-P., \& Véron, P. 2001, A\&A, 374, 92

Voges, W., Aschenbach, W., Boller, T., et al. 1999, A\&A, 349, 389

Voges, W., Aschenbach, W., Boller, T., et al. 2000, IAU Circ. 7432

Wampler, E. J., \& Ponz, D. 1985, ApJ, 298, 448

Williams, P. J. S., \& Bridle, A. H. 1967, The Observatory, 87, 280

Wisotzki, L., Christlieb, N., Bade, N., et al. 2000, A\&A, 358, 77

Zickgraf, F.-J., Thiering, I., Krautter, J., et al. 1997, A\&AS, 123, 103

Zickgraf, F.-J., Engels, D., Hagen, H.-J., Reimers, D. \& Voges, W. 2003, A\&A, 406, 535 
M.-P. Véron-Cetty et al.: Bright AGN in the ROSAT-FSC, Online Material $p 1$

\section{Online Material}


Table 2. List of the 185 galaxy candidates. Column 1: right ascension, Col. 2: declination, Col. 3: separation between the ROSAT and USNO positions in arcsec, Col. 4: X-ray count rate (count s ${ }^{-1}$ ), Col. 5: logarithm of the 0.1-2.4 keV X-ray luminosity (in erg s ${ }^{-1}$ ), Col. 6: magnitude $O_{\mathrm{USNO}}$, Col. 7: magnitude $O_{\mathrm{APS}}$, Col. 8: redshift, Col. 9: classification (abs: galaxies with absorption lines only; em: emission line galaxies, but too weak to make a classification possible; $\mathrm{C}$ stands for Composite spectrum; $S$ or $S$ ?: the object is certainly an AGN, but the lines are too weak for a more precise classification); Col. 10: references: (1) BAO; (2) OHP, Col. 11: name.

\begin{tabular}{|c|c|c|c|c|c|c|c|c|c|c|c|c|c|c|}
\hline \multicolumn{15}{|c|}{ J2000 optical position } \\
\hline $\mathrm{h}$ & $\mathrm{m}$ & $\mathrm{s}$ & & & & $\operatorname{sep}\left({ }^{\prime \prime}\right)$ & $c / r$ & $L_{\mathrm{X}}$ & $O_{\mathrm{US}}$ & $O_{\mathrm{APS}}$ & $z$ & & ref. & Name \\
\hline 0 & 14 & 7.46 & 10 & 34 & 13.3 & 5.3 & 0.036 & & 15.0 & 16.89 & & & & \\
\hline 0 & 19 & 37.80 & 29 & 56 & 2.0 & 27.2 & 0.042 & 42.02 & & 15.37 & 0.024 & & & NGC 76 \\
\hline 0 & 24 & 30.24 & 13 & 35 & 50.3 & 2.4 & 0.032 & & 12.5 & 16.63 & & & & NPM1G+13.0013 \\
\hline 0 & 46 & 29.20 & 8 & 25 & 59.6 & 18.9 & 0.023 & 42.18 & 15.5 & 16.32 & 0.039 & & & UGC 482 \\
\hline 0 & 54 & 45.23 & 16 & 26 & 17.2 & 9.2 & 0.038 & 42.37 & 13.6 & 16.56 & 0.038 & & & MCG+03.03.008 \\
\hline 0 & 54 & 48.64 & 28 & 52 & 1.1 & 26.7 & 0.024 & 41.74 & & 15.66 & 0.023 & & & UGC 555 \\
\hline 0 & 57 & 48.89 & 30 & 21 & 8.8 & 7.3 & 0.043 & 41.67 & & 13.14 & 0.016 & Liner & & NGC 315 \\
\hline 1 & 16 & 14.83 & 31 & 2 & 1.8 & 34.2 & 0.021 & 41.42 & & & 0.017 & & & NGC 452 \\
\hline 1 & 20 & 38.97 & 29 & 41 & 43.9 & 9.4 & 0.031 & 41.85 & 16.0 & 15.40 & 0.023 & & & IC 1672 \\
\hline 1 & 30 & 35.46 & 19 & 36 & 31.0 & 4.2 & 0.034 & 42.43 & 13.7 & 16.35 & 0.043 & & & UGC 1077 \\
\hline 2 & 43 & 48.71 & 14 & 53 & 13.1 & 33.8 & 0.027 & 42.39 & & 16.49 & 0.046 & & & IC 1835 \\
\hline 2 & 46 & 25.36 & 3 & 36 & 27.3 & 7.1 & 0.078 & 42.25 & 12.5 & 14.91 & 0.023 & & & NGC 1085 \\
\hline 2 & 51 & 12.98 & 13 & 11 & 31.3 & 16.1 & 0.031 & 41.92 & 13.8 & 16.34 & 0.025 & & & UGC 2337B \\
\hline 2 & 58 & 51.14 & 6 & 22 & 25.9 & 5.4 & 0.026 & 42.35 & 13.5 & 16.88 & 0.045 & & & LEDA 074274 \\
\hline 7 & 50 & 8.28 & 55 & 23 & 2.9 & 18.6 & 0.032 & 41.70 & 12.6 & 15.43 & 0.019 & abs & 2 & UGC 4035 \\
\hline 8 & 1 & 55.04 & 62 & 32 & 15.0 & 6.0 & 0.034 & 42.88 & 14.0 & & 0.072 & & & \\
\hline 8 & 30 & 29.14 & 69 & 1 & 42.8 & 12.3 & 0.022 & 41.66 & 13.5 & & 0.022 & & & Zw 331.048 \\
\hline 8 & 36 & 45.79 & 48 & 42 & 0.7 & 30.1 & 0.027 & & 13.7 & 16.24 & & & & MCG+08.16.019 \\
\hline 8 & 39 & 55.44 & 74 & 5 & 15.9 & 32.2 & 0.024 & & 15.1 & & & & & NPM1G+74.0038 \\
\hline 8 & 55 & 38.01 & 78 & 13 & 25.3 & 5.0 & 0.024 & 40.41 & & & 0.005 & Liner & & NGC 2655 \\
\hline 9 & 6 & 44.71 & 3 & 36 & 0.9 & 28.1 & 0.023 & & 14.2 & & & & & \\
\hline 9 & 8 & 38.35 & 32 & 35 & 34.0 & 15.9 & 0.033 & 41.44 & 12.4 & 15.79 & 0.014 & & & IC 2439 \\
\hline 9 & 11 & 37.50 & 60 & 2 & 15.0 & 22.9 & 0.026 & 40.45 & & 12.12 & 0.005 & $\mathrm{~S}$ & & NGC 2768 \\
\hline 9 & 12 & 38.04 & 75 & 38 & 55.4 & 13.2 & 0.040 & 42.81 & 12.8 & & 0.061 & H II & 1,2 & NPM1G+75.0038 \\
\hline 9 & 16 & 3.95 & 17 & 37 & 43.6 & 10.1 & 0.060 & 42.34 & & 14.63 & 0.029 & & & NGC 2795 \\
\hline 9 & 16 & 50.01 & 20 & 11 & 54.6 & 19.8 & 0.021 & 41.85 & & 15.04 & 0.028 & & & NGC 2804 \\
\hline 9 & 19 & 27.31 & 33 & 47 & 26.4 & 26.4 & 0.029 & 41.65 & 13.7 & 16.83 & 0.019 & & & LEDA 139185 \\
\hline 9 & 20 & 2.10 & 1 & 2 & 18.0 & 15.9 & 0.046 & 41.76 & & 14.27 & 0.017 & & & UGC 4956 \\
\hline 9 & 23 & 25.93 & 22 & 19 & 1.0 & 20.3 & 0.081 & 42.50 & 13.9 & & 0.030 & & & UGC 4991b \\
\hline 9 & 23 & 24.36 & 22 & 18 & 47.3 & 5.4 & 0.081 & 42.60 & 13.4 & & 0.034 & & & UGC 4991a \\
\hline 9 & 23 & 29.19 & 25 & 46 & 9.6 & 13.4 & 0.021 & 42.35 & 12.5 & 15.73 & 0.050 & S2 & 1,2 & Zw 121.071 \\
\hline 9 & 24 & 14.29 & 49 & 15 & 14.9 & 32.9 & 0.021 & 40.86 & & 14.71 & 0.009 & & & NGC 2856 \\
\hline 9 & 25 & 28.30 & 23 & 36 & 31.6 & 1.5 & 0.035 & 42.21 & 12.2 & 16.12 & 0.033 & $\mathrm{em}$ & 1 & $\mathrm{Zw} 121.086$ \\
\hline 9 & 25 & 42.55 & 11 & 25 & 55.3 & 14.6 & 0.034 & 41.25 & & 14.31 & 0.011 & & & NGC 2872 \\
\hline 9 & 32 & 10.13 & 21 & 30 & 4.4 & 18.1 & 0.030 & 39.71 & & & 0.002 & H II & & NGC 2903 \\
\hline 9 & 32 & 16.98 & 9 & 41 & 0.5 & 28.6 & 0.023 & & 14.8 & 16.74 & & & & NPM1G+09.0188 \\
\hline 9 & 38 & 12.30 & 7 & 43 & 39.8 & 19.9 & 0.033 & & 13.8 & 16.84 & & & & Zw 035.017 \\
\hline 9 & 38 & 32.90 & 17 & 1 & 52.0 & 17.9 & 0.040 & 42.13 & & 16.00 & 0.028 & & & NGC 2943 \\
\hline 9 & 45 & 28.84 & 56 & 32 & 53.4 & 2.1 & 0.046 & 43.58 & 15.8 & 16.91 & 0.139 & abs & 1 & \\
\hline 9 & 50 & 21.61 & 72 & 16 & 44.1 & 22.7 & 0.025 & 40.23 & & & 0.004 & S1.9 & & NGC 2985 \\
\hline 9 & 59 & 39.60 & 0 & 35 & 12.1 & 24.1 & 0.028 & 42.72 & 13.1 & 16.42 & 0.066 & & & UGC 5370 \\
\hline 10 & 1 & 57.80 & 55 & 40 & 47.1 & 4.2 & 0.049 & 40.53 & & 12.23 & 0.004 & S2 & & NGC 3079 \\
\hline 10 & 6 & 7.45 & 47 & 15 & 45.4 & 22.0 & 0.036 & 41.98 & & 15.09 & 0.025 & & & NGC 3111 \\
\hline 10 & 13 & 50.50 & 38 & 45 & 53.8 & 27.8 & 0.028 & 41.80 & & 14.15 & 0.023 & & & NGC 3158 \\
\hline 10 & 27 & 49.91 & 36 & 33 & 34.7 & 24.4 & 0.037 & 42.34 & & 16.02 & 0.037 & S2 & & HS $1024+3648$ \\
\hline 10 & 32 & 33.34 & 65 & 2 & 0.9 & 33.2 & 0.023 & 40.55 & & 14.42 & 0.006 & & & NGC 3259 \\
\hline 10 & 47 & 10.14 & 72 & 50 & 20.9 & 8.5 & 0.025 & 40.94 & & & 0.009 & & & NGC 3348 \\
\hline 10 & 51 & 29.91 & 46 & 44 & 41.5 & 24.1 & 0.026 & & 13.3 & 16.00 & & & & $\mathrm{NPM} 1 \mathrm{G}+47.0182$ \\
\hline 10 & 58 & 13.77 & 1 & 36 & 6.3 & 8.8 & 0.069 & 42.63 & 16.5 & & 0.038 & abs & 1 & UGC 6057c \\
\hline 10 & 58 & 13.04 & 1 & 36 & 24.3 & 29.9 & 0.069 & 42.65 & 15.1 & & 0.039 & & & UGC 6057b \\
\hline 11 & 0 & 5.35 & 14 & 50 & 26.5 & 28.4 & 0.026 & 40.45 & 15.9 & & 0.005 & & & NGC 3485 \\
\hline 11 & 3 & 11.35 & 27 & 58 & 21.1 & 7.8 & 0.034 & 40.56 & 12.2 & 13.02 & 0.005 & H II & & NGC 3504 \\
\hline 11 & 8 & 43.37 & 29 & 13 & 25.3 & 2.7 & 0.022 & 42.76 & 13.4 & 16.49 & 0.078 & em & 1,2 & LEDA 094150 \\
\hline 11 & 10 & 45.96 & 11 & 36 & 41.2 & 14.3 & 0.025 & 42.28 & 13.2 & 16.16 & 0.042 & S1 & 1 & Zw 067.027 \\
\hline
\end{tabular}


Table 2. continued.

\begin{tabular}{|c|c|c|c|c|c|c|c|c|c|c|c|c|c|c|}
\hline \multicolumn{15}{|c|}{ J2000 optical position } \\
\hline $\mathrm{h}$ & $\mathrm{m}$ & $\mathrm{s}$ & & & & $\operatorname{sep}\left({ }^{\prime \prime}\right)$ & $c / r$ & $L_{\mathrm{X}}$ & $O_{\mathrm{US}}$ & $O_{\mathrm{APS}}$ & $z$ & & ref. & Name \\
\hline 11 & 15 & 2.23 & 4 & 5 & 7.4 & 13.4 & 0.024 & 42.19 & 13.4 & 16.48 & 0.039 & & & Zw 039.090 \\
\hline 11 & 24 & 43.64 & 38 & 45 & 46.3 & 22.1 & 0.051 & 41.03 & 12.9 & 12.50 & 0.007 & H II & & NGC 3665 \\
\hline 11 & 25 & 45.33 & 24 & 8 & 24.5 & 28.9 & 0.029 & 41.86 & 12.7 & 15.12 & 0.024 & & & $\mathrm{Zw} 126.051$ \\
\hline 11 & 28 & 2.16 & 78 & 59 & 40.4 & 16.3 & 0.046 & & 14.7 & & 0.000 & H II & & UGC 6456 \\
\hline 11 & 34 & 6.64 & 25 & 33 & 34.8 & 18.9 & 0.029 & & 12.8 & 16.55 & & & & MCG+04.27.068 \\
\hline 11 & 39 & 14.88 & 17 & 8 & 37.2 & 4.2 & 0.037 & 41.85 & 13.1 & 16.23 & 0.021 & & & UGC 6614 \\
\hline 11 & 39 & 41.57 & 31 & 54 & 41.1 & 2.9 & 0.034 & 41.07 & 15.9 & & 0.009 & $\mathrm{~S} 1.8$ & & NGC 3786 \\
\hline 11 & 46 & 12.24 & 20 & 23 & 29.9 & 27.2 & 0.048 & 42.04 & 14.5 & 14.61 & 0.023 & Liner & & NGC 3884 \\
\hline 11 & 47 & 22.16 & 35 & 1 & 7.5 & 25.9 & 0.044 & 42.87 & 14.4 & 16.60 & 0.063 & S2 & & B2 1144+35B \\
\hline 11 & 49 & 59.31 & 21 & 20 & 1.1 & 9.2 & 0.026 & 41.88 & & 15.32 & 0.026 & & & NGC 3910 \\
\hline 11 & 50 & 41.53 & 20 & 0 & 54.6 & 10.2 & 0.031 & 41.77 & & 15.55 & 0.021 & & & NGC 3919 \\
\hline 11 & 53 & 26.26 & 35 & 56 & 53.8 & 15.3 & 0.023 & 42.66 & 12.3 & 16.27 & 0.068 & $\mathrm{~S} 2$ & 2 & KUG $1150+362$ \\
\hline 11 & 59 & 52.16 & 55 & 32 & 5.4 & 30.6 & 0.024 & 42.83 & 13.7 & 16.96 & 0.081 & & & PGC 2505536 \\
\hline 12 & 4 & 6.25 & 20 & 14 & 6.2 & 25.5 & 0.028 & 41.72 & 12.9 & 14.99 & 0.021 & & & NGC 4065 \\
\hline 12 & 4 & 43.34 & 31 & 10 & 38.2 & 16.7 & 0.025 & 41.83 & & 15.11 & 0.025 & S1.9 & & UGC 7064 \\
\hline 12 & 5 & 49.83 & 35 & 10 & 46.4 & 11.9 & 0.190 & 43.38 & 12.7 & 16.74 & 0.054 & $\mathrm{~S} 1$ & & Mark 646 \\
\hline 12 & 8 & 5.56 & 25 & 14 & 14.1 & 16.0 & 0.034 & 41.89 & & 15.32 & 0.023 & & & UGC 7115 \\
\hline 12 & 10 & 33.61 & 30 & 24 & 5.9 & 21.4 & 0.045 & 39.29 & & 13.71 & 0.001 & $\mathrm{C}$ & & NGC 4150 \\
\hline 12 & 14 & 18.10 & 29 & 31 & 4.5 & 5.0 & 0.021 & 42.57 & & 16.96 & 0.064 & $\mathrm{~S} 2$ & & WAS 49b \\
\hline 12 & 14 & 48.65 & 59 & 54 & 22.5 & 23.3 & 0.028 & 42.64 & 14.6 & 15.61 & 0.060 & & & NGC 4199a \\
\hline 12 & 14 & 51.71 & 59 & 54 & 30.5 & 4.5 & 0.028 & 42.65 & 16.3 & & 0.061 & abs & 1 & NGC 4199b \\
\hline 12 & 15 & 5.34 & 76 & 14 & 10.0 & 34.4 & 0.031 & 40.68 & & & 0.006 & & & UGC 7265 \\
\hline 12 & 16 & 0.00 & 12 & 41 & 1.4 & 5.4 & 0.033 & 42.78 & & 16.60 & 0.065 & S1.9 & & Mark 764 \\
\hline 12 & 19 & 51.66 & 28 & 25 & 21.7 & 12.6 & 0.046 & 42.13 & 13.8 & 16.27 & 0.026 & & & $\mathrm{Zw} 158.075$ \\
\hline 12 & 20 & 6.86 & 29 & 16 & 50.5 & 11.4 & 0.037 & 39.80 & 12.6 & 12.53 & 0.002 & Liner & & NGC 4278 \\
\hline 12 & 22 & 54.89 & 15 & 49 & 20.7 & 15.6 & 0.042 & 40.65 & & 11.30 & 0.005 & $\mathrm{C}$ & & NGC 4321 \\
\hline 12 & 23 & 39.10 & 7 & 3 & 14.0 & 24.6 & 0.030 & 40.06 & & 14.80 & 0.003 & & & NGC 4342 \\
\hline 12 & 23 & 42.85 & 58 & 14 & 45.9 & 10.3 & 0.037 & 41.55 & 14.5 & 16.93 & 0.015 & $\mathrm{~S}$ ? & & SBS $1221+585$ \\
\hline 12 & 26 & 28.02 & 9 & 1 & 23.0 & 26.7 & 0.029 & 41.86 & 14.7 & 14.58 & 0.024 & Liner & & NGC 4410 \\
\hline 12 & 26 & 26.98 & 31 & 13 & 22.6 & 4.4 & 0.033 & 39.75 & 12.2 & 12.17 & 0.002 & $\mathrm{C}$ & & NGC 4414 \\
\hline 12 & 28 & 29.49 & 17 & 5 & 6.0 & 9.8 & 0.039 & 40.91 & & 12.21 & 0.007 & Liner & & NGC 4450 \\
\hline 12 & 34 & 3.10 & 7 & 41 & 59.0 & 24.2 & 0.032 & 39.14 & & 11.85 & 0.001 & H II & & NGC 4526 \\
\hline 12 & 34 & 46.81 & 47 & 45 & 32.4 & 34.2 & 0.043 & 42.25 & & 16.12 & 0.031 & & & MCG+08.23.061 \\
\hline 12 & 48 & 58.34 & 40 & 35 & 56.8 & 14.5 & 0.039 & 41.58 & 15.2 & 15.95 & 0.015 & & & IC 3808 \\
\hline 12 & 50 & 26.60 & 25 & 30 & 5.8 & 8.3 & 0.028 & 40.28 & & 11.04 & 0.004 & $\mathrm{~S} 2$ & & NGC 4725 \\
\hline 12 & 55 & 7.78 & 78 & 37 & 14.9 & 4.1 & 0.029 & 42.36 & 12.9 & & 0.043 & $\mathrm{~S} 1$ & 1 & NPM1G+78.0053 \\
\hline 12 & 56 & 43.76 & 21 & 40 & 51.9 & 7.2 & 0.041 & 39.25 & & 10.36 & 0.001 & $\mathrm{~S}$ & & NGC 4826 \\
\hline 12 & 59 & 39.35 & 38 & 48 & 56.3 & 16.0 & 0.022 & 42.01 & 13.2 & 16.23 & 0.033 & & & IC 4003 \\
\hline 13 & 0 & 39.13 & 2 & 30 & 5.3 & 22.4 & 0.027 & 40.02 & & 12.98 & 0.003 & H II & & NGC 4900 \\
\hline 13 & 4 & 40.89 & 43 & 18 & 35.0 & 20.1 & 0.031 & & 14.0 & 16.86 & & & & $\mathrm{NPM} 1 \mathrm{G}+43.0235$ \\
\hline 13 & 4 & 57.99 & 43 & 33 & 10.9 & 25.2 & 0.052 & & 13.1 & 16.06 & & & & $\mathrm{MCG}+07.27 .026$ \\
\hline 13 & 7 & 3.03 & 56 & 31 & 59.2 & 5.5 & 0.044 & 43.08 & 12.4 & 16.40 & 0.080 & S1 & 1,2 & MCG+10.19.033 \\
\hline 13 & 20 & 28.94 & 31 & 21 & 6.9 & 19.8 & 0.029 & 42.42 & 12.8 & 16.53 & 0.046 & & & Zw 160.204 \\
\hline 13 & 29 & 58.73 & 47 & 16 & 4.5 & 29.1 & 0.046 & 39.90 & & 11.51 & 0.002 & Liner & & NGC 5195 \\
\hline 13 & 32 & 48.62 & 41 & 52 & 18.9 & 12.3 & 0.038 & 42.07 & & 14.08 & 0.027 & & & NGC 5214 \\
\hline 13 & 38 & 17.27 & 48 & 16 & 32.0 & 18.4 & 0.043 & 42.16 & & 15.05 & 0.028 & $\mathrm{~S} 2$ & & Mark 266SW \\
\hline 13 & 42 & 8.34 & 35 & 39 & 15.2 & 15.3 & 0.021 & 40.16 & & 13.06 & 0.004 & S1.9 & & NGC 5273 \\
\hline 13 & 44 & 1.90 & 25 & 56 & 27.8 & 28.5 & 0.026 & & 12.8 & 17.00 & & & & \\
\hline 13 & 51 & 4.24 & 19 & 26 & 8.2 & 3.7 & 0.048 & 42.90 & 13.5 & 16.95 & 0.062 & $\mathrm{em}$ & 1,2 & \\
\hline 13 & 51 & 42.19 & 55 & 59 & 43.2 & 29.3 & 0.022 & & 15.1 & 16.68 & & & & TEX $1349+562$ \\
\hline 13 & 53 & 9.75 & 24 & 22 & 37.4 & 6.6 & 0.027 & 42.26 & 13.5 & 16.85 & 0.056 & $\mathrm{~S} 1$ & 1,2 & Zw 132.035 \\
\hline 13 & 53 & 26.69 & 40 & 16 & 58.9 & 7.7 & 0.048 & 41.12 & & & 0.008 & Liner & & NGC 5353 \\
\hline 13 & 53 & 43.82 & 33 & 13 & 20.3 & 28.2 & 0.024 & 42.43 & 14.5 & 15.61 & 0.051 & & & $\mathrm{MCG}+06.31 .015$ \\
\hline 13 & 53 & 55.94 & 21 & 59 & 54.9 & 24.1 & 0.042 & & 12.9 & 16.86 & & & & \\
\hline 13 & 58 & 19.76 & 7 & 13 & 15.2 & 27.4 & 0.044 & 42.07 & 15.7 & & 0.025 & & & Zw 046.025 \\
\hline 14 & 12 & 36.95 & 39 & 18 & 53.8 & 21.2 & 0.034 & 41.96 & 16.4 & 15.27 & 0.025 & S1.9 & & NGC 5515 \\
\hline 14 & 13 & 23.13 & 24 & 31 & 56.8 & 24.3 & 0.024 & 42.44 & 12.2 & 16.34 & 0.052 & abs & 2 & $\mathrm{Zw} 133.012$ \\
\hline 14 & 18 & 9.20 & 7 & 33 & 52.0 & 24.3 & 0.042 & 42.02 & & 14.97 & 0.024 & & & NGC 5546 \\
\hline
\end{tabular}


Table 2. continued.

\begin{tabular}{|c|c|c|c|c|c|c|c|c|c|c|c|c|c|c|}
\hline \multicolumn{15}{|c|}{$\overline{\text { J2000 optical position }}$} \\
\hline $\mathrm{h}$ & $\mathrm{m}$ & $\mathrm{s}$ & & & & $\operatorname{sep}\left({ }^{\prime \prime}\right)$ & $c / r$ & $L_{\mathrm{X}}$ & $O_{\mathrm{US}}$ & $O_{\mathrm{APS}}$ & $z$ & & ref. & Name \\
\hline 14 & 22 & 55.37 & 32 & 51 & 2.7 & 2.0 & 0.028 & 42.14 & & 15.28 & 0.034 & S1.8 & & UGC 9214 \\
\hline 14 & 26 & 2.32 & 8 & 6 & 40.5 & 34.6 & 0.024 & 41.84 & & 16.98 & 0.026 & & & $\mathrm{Zw} 047.039$ \\
\hline 14 & 26 & 18.64 & 26 & 14 & 52.3 & 13.8 & 0.027 & 42.02 & 16.3 & 15.58 & 0.030 & & & IC 4423 \\
\hline 14 & 28 & 23.06 & 78 & 53 & 8.2 & 0.2 & 0.047 & 42.03 & 12.1 & & 0.023 & & & IC 4470 \\
\hline 14 & 29 & 40.64 & 0 & 21 & 59.2 & 13.8 & 0.025 & 42.51 & 14.8 & 16.92 & 0.055 & abs & 1 & Zw 019.045 \\
\hline 14 & 29 & 48.51 & 53 & 57 & 54.3 & 25.6 & 0.025 & 42.30 & 13.2 & 16.19 & 0.043 & & & IC 1027 \\
\hline 14 & 42 & 39.65 & 29 & 20 & 48.2 & 7.5 & 0.028 & 42.82 & 13.5 & 17.00 & 0.074 & H II & 1,2 & NPM1G+29.0326 \\
\hline 14 & 50 & 51.40 & 5 & 6 & 52.0 & 25.9 & 0.025 & 41.92 & & 15.29 & 0.028 & S2 & & NGC 5765B \\
\hline 14 & 50 & 55.42 & 27 & 34 & 42.1 & 13.1 & 0.032 & 42.09 & & 15.89 & 0.030 & & & IC 4514 \\
\hline 14 & 51 & 14.39 & 30 & 41 & 32.2 & 33.9 & 0.044 & & 12.5 & 16.28 & & & & $\mathrm{Zw} 164.036$ \\
\hline 14 & 55 & 28.21 & 32 & 50 & 24.0 & 30.3 & 0.026 & & 14.1 & 16.21 & & & & MCG+06.33.009 \\
\hline 15 & 4 & 15.88 & 28 & 29 & 47.5 & 32.7 & 0.127 & 43.26 & & 15.66 & 0.058 & & & $\mathrm{MCG}+05.36 .002$ \\
\hline 15 & 5 & 56.60 & 3 & 42 & 26.0 & 15.6 & 0.027 & 42.18 & & 16.00 & 0.036 & S1.8 & & Mark 1392 \\
\hline 15 & 9 & 20.51 & 7 & 38 & 19.4 & 28.5 & 0.044 & 43.05 & 16.0 & 16.94 & 0.077 & abs & 2 & \\
\hline 15 & 13 & 40.25 & 4 & 4 & 17.2 & 24.9 & 0.025 & 42.21 & 13.3 & 16.96 & 0.039 & H II & 2 & Zw 049.061 \\
\hline 15 & 20 & 29.00 & 44 & 58 & 15.3 & 11.5 & 0.046 & 42.89 & 13.9 & 16.86 & 0.063 & abs & 1 & \\
\hline 15 & 21 & 20.58 & 30 & 40 & 15.3 & 15.0 & 0.139 & 43.57 & 14.0 & 16.93 & 0.079 & abs & 2 & $\mathrm{Zw} 165.041$ \\
\hline 15 & 23 & 59.90 & 31 & 12 & 40.2 & 31.4 & 0.032 & 42.97 & & 16.71 & 0.074 & & & MCG+05.36.026 \\
\hline 15 & 25 & 8.75 & 12 & 52 & 57.5 & 24.6 & 0.039 & 41.95 & 12.5 & 16.30 & 0.023 & & & Zw 077.123 \\
\hline 15 & 29 & 14.68 & 52 & 51 & 50.1 & 12.9 & 0.029 & 42.80 & 13.1 & 16.66 & 0.071 & abs & 1 & UGC 9868 \\
\hline 15 & 32 & 32.02 & 4 & 40 & 51.4 & 13.8 & 0.048 & 42.50 & & 15.75 & 0.039 & & & UGC 9886 \\
\hline 15 & 32 & 57.19 & 0 & 26 & 36.1 & 11.4 & 0.044 & 42.41 & 15.8 & 16.11 & 0.037 & & & $\mathrm{Zw} 022.010$ \\
\hline 15 & 33 & 17.73 & 82 & 13 & 46.2 & 11.3 & 0.036 & 41.87 & 12.9 & & 0.022 & & & UGC 9950 \\
\hline 15 & 35 & 54.26 & 14 & 31 & 2.7 & 34.6 & 0.049 & 41.92 & & 15.70 & 0.020 & $\mathrm{~S} 2$ & & Akn 479 \\
\hline 15 & 38 & 10.00 & 57 & 36 & 12.0 & 12.1 & 0.034 & 42.90 & & 16.20 & 0.074 & $\mathrm{~S} 1$ & & $\mathrm{MCG}+10.22 .028$ \\
\hline 15 & 39 & 2.65 & 31 & 45 & 34.3 & 25.4 & 0.031 & 40.81 & & 15.31 & 0.007 & & & NGC 5974 \\
\hline 15 & 47 & 48.93 & 37 & 1 & 36.0 & 19.9 & 0.040 & 43.01 & 15.1 & 16.60 & 0.077 & $\mathrm{em}$ & 2 & $\mathrm{NPM} 1 \mathrm{G}+37.0489$ \\
\hline 15 & 52 & 12.04 & 34 & 5 & 35.4 & 24.0 & 0.024 & 42.43 & 14.7 & & 0.051 & abs & 2 & $\mathrm{NPM} 1 \mathrm{G}+34.0353$ \\
\hline 15 & 56 & 41.39 & 20 & 10 & 16.4 & 33.0 & 0.027 & & 12.8 & 16.66 & & & & \\
\hline 16 & 4 & 55.53 & 28 & 9 & 56.9 & 21.8 & 0.047 & 43.08 & 12.1 & 16.54 & 0.077 & & & $\mathrm{Zw} 167.022$ \\
\hline 16 & 5 & 29.18 & 16 & 25 & 8.8 & 28.3 & 0.041 & 42.53 & & & 0.044 & & & UGC 10187B \\
\hline 16 & 5 & 35.57 & 44 & 12 & 22.0 & 16.9 & 0.056 & 42.65 & 13.5 & 16.33 & 0.043 & & & $\mathrm{MCG}+07.33 .038$ \\
\hline 16 & 5 & 36.83 & 17 & 48 & 8.1 & 32.0 & 0.025 & 42.09 & & 16.32 & 0.034 & H II & & Mark 298 \\
\hline 16 & 7 & 24.03 & 85 & 1 & 49.3 & 2.7 & 0.042 & 43.78 & 16.5 & & 0.183 & $\mathrm{~S} 1$ & & S5 $1616+85$ \\
\hline 16 & 7 & 35.36 & 13 & 56 & 37.6 & 28.9 & 0.026 & 42.11 & 13.1 & 16.21 & 0.034 & & & NGC 6066 \\
\hline 16 & 9 & 5.53 & 27 & 53 & 34.0 & 10.2 & 0.041 & 42.23 & 12.5 & 16.84 & 0.031 & $\mathrm{~S} 2$ & 1,2 & NPM1G+28.0373 \\
\hline 16 & 9 & 35.18 & 63 & 58 & 1.2 & 8.2 & 0.047 & 42.75 & 14.0 & 16.82 & 0.053 & $\mathrm{em}$ & 1,2 & NPM1G+64.0143 \\
\hline 16 & 11 & 11.40 & 61 & 16 & 4.5 & 13.2 & 0.030 & 42.09 & & 14.95 & 0.031 & & & NGC 6095 \\
\hline 16 & 11 & 13.89 & 36 & 58 & 24.3 & 27.6 & 0.031 & 42.79 & 12.5 & 16.95 & 0.068 & abs & 2 & KUG $1609+371 \mathrm{~A}$ \\
\hline 16 & 12 & 33.72 & 29 & 29 & 39.1 & 15.5 & 0.043 & 42.28 & 13.6 & 15.07 & 0.032 & & & NGC 6086 \\
\hline 16 & 24 & 37.00 & 19 & 30 & 24.3 & 11.2 & 0.026 & 42.16 & & & 0.036 & & & Zw 109.013 \\
\hline 16 & 27 & 42.60 & 39 & 22 & 38.5 & 13.7 & 0.023 & 41.92 & 16.1 & & 0.029 & & & PGC 058195 \\
\hline 16 & 29 & 44.90 & 40 & 48 & 41.8 & 6.2 & 0.039 & 42.15 & & 14.31 & 0.029 & & & NGC 6173 \\
\hline 16 & 29 & 52.80 & 24 & 26 & 38.1 & 6.3 & 0.041 & 42.40 & 12.2 & 16.30 & 0.038 & S1.9 & & Mark 883 \\
\hline 16 & 32 & 58.03 & 11 & 43 & 23.8 & 27.5 & 0.038 & 42.71 & 16.3 & 15.65 & 0.056 & & & Zw 080.046 \\
\hline 16 & 33 & 48.87 & 35 & 53 & 18.8 & 33.3 & 0.022 & 42.13 & & 16.57 & 0.038 & & & KUG $1632+359$ \\
\hline 16 & 36 & 57.72 & 55 & 46 & 58.2 & 8.6 & 0.038 & 42.11 & 13.5 & 16.92 & 0.028 & $\mathrm{~S}$ ? & 1,2 & \\
\hline 16 & 37 & 20.55 & 41 & 11 & 20.1 & 16.3 & 0.038 & 42.22 & 13.0 & 16.74 & 0.032 & abs & 2 & NPM1G+41.0441 \\
\hline 16 & 39 & 4.72 & 8 & 21 & 30.9 & 11.2 & 0.035 & 42.48 & 13.2 & 16.88 & 0.045 & $\mathrm{em}$ & 2 & $\mathrm{NPM} 1 \mathrm{G}+08.0448$ \\
\hline 16 & 42 & 56.33 & 19 & 15 & 15.5 & 10.6 & 0.042 & 42.32 & 13.0 & 16.25 & 0.034 & & & IC 1224 \\
\hline 16 & 43 & 4.22 & 61 & 34 & 43.3 & 22.7 & 0.032 & 41.70 & 13.9 & 14.13 & 0.019 & & & NGC 6223 \\
\hline 16 & 56 & 1.58 & 21 & 12 & 42.0 & 10.4 & 0.028 & 42.46 & 15.4 & 16.99 & 0.049 & S1 & 1 & NPM1G+21.0507 \\
\hline 16 & 57 & 45.01 & 68 & 30 & 53.1 & 13.9 & 0.045 & 42.42 & 13.1 & 15.88 & 0.037 & & & NGC 6289 \\
\hline 17 & 0 & 27.21 & 51 & 59 & 11.6 & 14.3 & 0.044 & 42.64 & 12.7 & 16.87 & 0.048 & S1 & & $\mathrm{NPM} 1 \mathrm{G}+52.0273$ \\
\hline 17 & 3 & 47.89 & 34 & 43 & 39.2 & 20.0 & 0.023 & & 12.2 & & & & & \\
\hline 17 & 12 & 36.58 & 38 & 1 & 13.3 & 25.4 & 0.040 & 42.37 & & 15.40 & 0.037 & & & IC 1245 \\
\hline 17 & 15 & 58.85 & 36 & 23 & 23.1 & 15.2 & 0.048 & 43.18 & 13.6 & 16.13 & 0.086 & S1 & 2 & UGC 10782 \\
\hline 17 & 23 & 22.08 & 32 & 49 & 55.2 & 16.7 & 0.022 & 42.63 & 13.2 & 16.55 & 0.067 & H II & 2 & \\
\hline
\end{tabular}


M.-P. Véron-Cetty et al.: Bright AGN in the ROSAT-FSC, Online Material p 5

Table 2. continued.

\begin{tabular}{|c|c|c|c|c|c|c|c|c|c|c|c|c|c|c|}
\hline \multicolumn{15}{|c|}{ J2000 optical position } \\
\hline $\mathrm{h}$ & $\mathrm{m}$ & $\mathrm{s}$ & & & & $\operatorname{sep}\left({ }^{\prime \prime}\right)$ & $c / r$ & $L_{\mathrm{X}}$ & $O_{\mathrm{US}}$ & $O_{\mathrm{APS}}$ & $z$ & & ref. & Name \\
\hline 17 & 37 & 56.34 & 41 & 38 & 32.0 & 19.9 & 0.023 & 42.68 & 15.1 & 16.39 & 0.070 & H II & 2 & IRAS F17363+4140 \\
\hline 17 & 38 & 11.38 & 58 & 42 & 55.3 & 21.7 & 0.034 & 42.09 & & 15.85 & 0.029 & S1 & & NGC 6418 \\
\hline 22 & 21 & 47.39 & 2 & 54 & 36.2 & 24.5 & 0.028 & & 13.2 & 16.72 & & & & \\
\hline 22 & 28 & 29.51 & 16 & 46 & 59.6 & 11.6 & 0.034 & 42.23 & 12.9 & 15.01 & 0.034 & & & NGC 7291 \\
\hline 22 & 32 & 30.68 & 8 & 12 & 33.0 & 18.2 & 0.034 & 41.96 & 12.5 & 16.08 & 0.025 & S1 & & Akn 557 \\
\hline 22 & 35 & 40.82 & 1 & 29 & 5.9 & 26.2 & 0.293 & 43.64 & 14.3 & 16.63 & 0.059 & & & LEDA 087323 \\
\hline 22 & 41 & 34.22 & 4 & 53 & 10.7 & 34.3 & 0.047 & 42.97 & & 16.90 & 0.068 & $\mathrm{~S}$ ? & & NPM1G+04.0574 \\
\hline 22 & 49 & 54.69 & 11 & 36 & 30.1 & 23.7 & 0.054 & 42.19 & & 14.35 & 0.026 & & & NGC 7385 \\
\hline 22 & 58 & 1.97 & 13 & 8 & 4.4 & 10.7 & 0.021 & 41.75 & & 15.08 & 0.025 & & & NGC 7432 \\
\hline 23 & 13 & 58.36 & 3 & 42 & 54.4 & 5.6 & 0.031 & 42.62 & 13.0 & 16.42 & 0.056 & & & Zw 380.012 \\
\hline 23 & 20 & 42.29 & 8 & 13 & 2.5 & 7.2 & 0.035 & 41.26 & & & 0.011 & Liner & & NGC 7626 \\
\hline 23 & 25 & 51.50 & 8 & 47 & 11.2 & 6.6 & 0.025 & & 14.3 & 16.46 & & & & KUG $2323+085$ \\
\hline 23 & 31 & 50.20 & 25 & 32 & 40.0 & 6.7 & 0.038 & & 13.3 & 16.59 & & & & KUG $2329+252$ \\
\hline 23 & 36 & 14.10 & 2 & 9 & 18.6 & 3.5 & 0.021 & 40.86 & & & 0.009 & H II & & NGC 7714 \\
\hline 23 & 56 & 1.96 & 7 & 31 & 23.4 & 3.1 & 0.049 & 42.53 & 13.6 & 16.84 & 0.040 & S1 & & Mark 541 \\
\hline
\end{tabular}


Table 3. List of the 76 known AGN brighter than $O_{\mathrm{USNO}}=16.5$, located within $35^{\prime \prime}$ of a ROSAT-FSC source. Column 1: right ascension, Col. 2: declination, Col. 3: separation between the ROSAT and USNO positions in arcsec, Col. 4: X-ray count rate (count s $\mathrm{s}^{-1}$ ), Col. 5: magnitude $O_{\mathrm{USNO}}$, Col. 6: magnitude $O_{\mathrm{APS}}$, Col. 7: APS $O-E$ colour, Col. 8: redshift, Col. 9: absolute magnitude computed by using the $O_{\mathrm{USNO}}$ magnitude $\left(H_{\mathrm{o}}=50 \mathrm{~km} \mathrm{~s}^{-1} \mathrm{Mpc}^{-1}\right)$, Col. 10: classification, Col. 11: name.

\begin{tabular}{|c|c|c|c|c|c|c|c|c|c|c|c|c|c|c|}
\hline \multicolumn{15}{|c|}{ J2000 USNO position } \\
\hline $\bar{h}$ & $\mathrm{~m}$ & $\mathrm{~s}$ & & & & & $c / r$ & $O_{\mathrm{US}}$ & $O_{\mathrm{APS}}$ & $O-E$ & $z$ & $M_{\mathrm{abs}}$ & & Name \\
\hline 0 & 6 & 23.08 & 12 & 35 & 53.2 & 20.8 & 0.030 & 15.8 & 16.26 & 0.36 & 0.98 & -28.4 & $\mathrm{Q}$ & RGB J0006+125 \\
\hline 0 & 24 & 44.10 & 0 & 32 & 21.4 & 9.7 & 0.047 & 16.2 & 16.92 & 0.58 & 0.404 & -25.8 & $\mathrm{Q}$ & PB 5932 \\
\hline 0 & 36 & 12.50 & 5 & 49 & 52.0 & 9.1 & 0.064 & 16.3 & 16.88 & 0.28 & 0.41 & -25.7 & $\mathrm{Q}$ & HS $0033+0533$ \\
\hline 0 & 52 & 5.57 & 0 & 35 & 38.4 & 28.6 & 0.024 & 16.3 & 16.80 & 1.04 & 0.399 & -25.7 & $\mathrm{Q}$ & Q 0049+0019A \\
\hline 0 & 57 & 9.93 & 14 & 46 & 10.4 & 26.7 & 0.246 & 15.9 & 16.11 & 1.12 & 0.171 & -24.0 & $\mathrm{Q}$ & PHL 909 \\
\hline 1 & 20 & 17.29 & 21 & 33 & 46.6 & 25.0 & 0.023 & 15.5 & 15.80 & 0.54 & 1.500 & -29.7 & Q & PG $0117+213$ \\
\hline 1 & 39 & 55.80 & 6 & 19 & 22.5 & 8.4 & 0.031 & 15.7 & 16.55 & 0.60 & 0.396 & -26.5 & $\mathrm{Q}$ & PHL 1092 \\
\hline 1 & 40 & 35.02 & 23 & 44 & 51.1 & 25.1 & 0.029 & 16.1 & 16.64 & 0.98 & 0.32 & -25.4 & $\mathrm{Q}$ & HS $0137+2329$ \\
\hline 1 & 41 & 59.60 & 6 & 12 & 5.5 & 2.7 & 0.049 & 16.3 & 16.27 & 0.56 & 0.345 & -27.2 & $\mathrm{Q}$ & PHL 1106 \\
\hline 3 & 18 & 25.57 & 15 & 59 & 56.7 & 11.2 & 0.033 & 16.3 & 16.58 & 0.02 & 0.515 & -25.9 & $\mathrm{Q}$ & US 3828 \\
\hline 3 & 39 & 9.60 & 3 & 45 & 52.5 & 23.7 & 0.038 & 16.4 & 17.55 & 0.44 & 0.199 & -24.0 & Q & RXS J03391+0346 \\
\hline 4 & 24 & 46.85 & 0 & 36 & 6.4 & 7.4 & 0.052 & 16.1 & 16.75 & 1.26 & & & BL & PKS 0422+00 \\
\hline 8 & 15 & 59.21 & 41 & 44 & 57.1 & 17.9 & 0.021 & 15.8 & 15.97 & 0.32 & 1.28 & -29.3 & Q & KUV 08126+4154 \\
\hline 8 & 22 & 36.88 & 54 & 18 & 36.4 & 33.7 & 0.042 & 16.3 & 16.65 & 1.12 & 0.086 & -22.3 & S1 & SBS $0818+544$ \\
\hline 8 & 43 & 49.78 & 26 & 19 & 11.1 & 19.4 & 0.068 & 16.4 & 17.23 & 0.20 & 0.258 & -24.7 & Q & HS $0840+2630$ \\
\hline 8 & 59 & 24.35 & 46 & 37 & 17.3 & 22.3 & 0.047 & 16.5 & 16.89 & 0.74 & 0.923 & -27.5 & $\mathrm{Q}$ & US 2068 \\
\hline 9 & 0 & 47.30 & 74 & 44 & 26.3 & 16.6 & 0.045 & 16.1 & & & 0.77 & -27.4 & $\mathrm{Q}$ & HS $0855+7456$ \\
\hline 9 & 19 & 57.63 & 51 & 6 & 9.1 & 17.1 & 0.039 & 15.8 & 16.35 & -0.80 & 0.553 & -27.0 & $\mathrm{Q}$ & SBS $0916+513$ \\
\hline 9 & 25 & 14.35 & 54 & 44 & 27.2 & 10.4 & 0.048 & 15.9 & 16.66 & -0.12 & 0.476 & -26.3 & $\mathrm{Q}$ & SBS $0921+549$ \\
\hline 9 & 29 & 9.82 & 46 & 44 & 24.1 & 15.9 & 0.025 & 15.6 & 15.66 & 0.18 & 0.240 & -25.3 & $\mathrm{Q}$ & US 645 \\
\hline 9 & 36 & 25.45 & 39 & 49 & 33.7 & 24.8 & 0.033 & 16.1 & 16.81 & 0.82 & 1.25 & -28.7 & Q & KUV 09333+4003 \\
\hline 9 & 37 & 1.93 & 34 & 25 & 0.0 & 12.7 & 0.031 & 16.0 & 16.55 & 0.22 & 0.908 & -28.1 & $\mathrm{Q}$ & Ton 1078 \\
\hline 9 & 41 & 33.72 & 59 & 48 & 11.3 & 13.2 & 0.045 & 16.3 & 16.70 & 0.36 & 0.966 & -27.9 & $\mathrm{Q}$ & SBS $0938+600$ \\
\hline 9 & 56 & 49.89 & 25 & 15 & 16.0 & 8.0 & 0.048 & 16.2 & 16.92 & 0.84 & 0.712 & -27.2 & $\mathrm{Q}$ & OK 290 \\
\hline 10 & 4 & 20.10 & 5 & 13 & 0.6 & 22.1 & 0.021 & 16.2 & 16.55 & 0.26 & 0.161 & -23.8 & $\mathrm{Q}$ & PG 1001+054 \\
\hline 10 & 13 & 30.20 & 53 & 15 & 59.6 & 6.7 & 0.025 & 16.2 & 16.64 & 0.36 & 1.495 & -29.1 & $\mathrm{Q}$ & SBS $1010+535$ \\
\hline 10 & 15 & 57.04 & 1 & 9 & 13.7 & 16.1 & 0.082 & 16.3 & 16.97 & 0.94 & 0.779 & -27.2 & $\mathrm{Q}$ & Q 1013+0124 \\
\hline 10 & 33 & 59.50 & 35 & 55 & 9.0 & 14.9 & 0.046 & 16.3 & 16.76 & 0.92 & 0.169 & -23.8 & Q & CSO 275 \\
\hline 10 & 43 & 55.52 & 56 & 27 & 57.0 & 18.6 & 0.028 & 16.3 & 16.81 & -0.40 & 1.951 & -29.6 & $\mathrm{Q}$ & SBS $1040+567$ \\
\hline 11 & 17 & 6.41 & 44 & 13 & 33.8 & 8.1 & 0.023 & 14.8 & 15.06 & 0.40 & 0.144 & -24.9 & $\mathrm{Q}$ & PG 1114+445 \\
\hline 11 & 30 & 4.76 & 41 & 16 & 19.5 & 23.2 & 0.035 & 15.7 & 16.20 & 0.10 & 1.530 & -29.5 & Q & KUV 11274+4133 \\
\hline 11 & 33 & 35.40 & 9 & 39 & 1.8 & 24.6 & 0.029 & 16.4 & 17.05 & 0.06 & 0.379 & -25.5 & $\mathrm{Q}$ & $5+0939$ \\
\hline 11 & 43 & 47.71 & 11 & 28 & 48.1 & 20.6 & 0.046 & 15.5 & 16.38 & 0.80 & 0.118 & -23.8 & $\mathrm{Q}$ & RX J11437+1128 \\
\hline 11 & 52 & 51.90 & 33 & 7 & 18.8 & 2.7 & 0.033 & 16.1 & 16.30 & 0.40 & 1.389 & -28.8 & Q & CSO 373 \\
\hline 11 & 55 & 7.62 & 52 & 1 & 29.4 & 6.4 & 0.036 & 16.4 & 16.46 & 1.08 & 0.156 & -23.5 & $\mathrm{Q}$ & SBS $1152+523$ \\
\hline 11 & 59 & 6.78 & 53 & 6 & 43.5 & 6.1 & 0.043 & 16.5 & 17.19 & 0.36 & 0.482 & -25.9 & $\mathrm{Q}$ & MS $11565+5323$ \\
\hline 12 & 7 & 4.53 & 38 & 40 & 24.6 & 3.7 & 0.045 & 16.3 & 16.64 & 0.00 & 0.572 & -26.4 & $\mathrm{Q}$ & RXS J12070+3840 \\
\hline 12 & 17 & 40.83 & 49 & 31 & 17.9 & 1.1 & 0.049 & 16.5 & 17.62 & 0.18 & 0.730 & -26.6 & Q & SBS 1215+497 \\
\hline 12 & 22 & 10.01 & 27 & 19 & 2.3 & 6.4 & 0.046 & 15.9 & 16.90 & 0.52 & 0.442 & -26.3 & $\mathrm{Q}$ & RXS J12221+2719 \\
\hline 12 & 23 & 0.23 & 55 & 40 & 0.3 & 9.4 & 0.057 & 16.5 & 17.48 & 0.48 & 0.905 & -27.5 & $\mathrm{Q}$ & SBS 1220+559 \\
\hline 12 & 28 & 24.97 & 31 & 28 & 37.7 & 24.8 & 0.024 & 15.6 & 16.28 & 0.28 & 2.219 & -30.4 & Q & B2 1225+31 \\
\hline 12 & 30 & 50.04 & 1 & 15 & 22.6 & 6.4 & 0.041 & 14.4 & 14.81 & 0.16 & 0.117 & -24.8 & Q & RX J12308+0115 \\
\hline 12 & 33 & 26.05 & 45 & 12 & 23.1 & 11.2 & 0.032 & 16.5 & 16.91 & -0.24 & 1.958 & -29.4 & Q & HS 1231+4528 \\
\hline 12 & 44 & 10.82 & 17 & 21 & 4.5 & 16.3 & 0.027 & 15.7 & 16.58 & 0.74 & 1.283 & -28.8 & $\mathrm{Q}$ & $1+176$ \\
\hline 13 & 6 & 5.72 & 80 & 8 & 20.5 & 10.9 & 0.035 & 16.3 & 18.35 & 1.01 & 1.183 & -28.3 & $\mathrm{Q}$ & S5 130 \\
\hline 13 & 13 & 21.39 & 78 & 21 & 53.9 & 11.9 & 0.026 & 15.6 & 15.86 & 0.22 & 2.00 & -30.3 & Q & HS $1312+7837$ \\
\hline 13 & 19 & 56.24 & 27 & 28 & 8.4 & 23.4 & 0.031 & 15.5 & 16.54 & 0.22 & 1.014 & -28.7 & Q & CSO 873 \\
\hline 13 & 41 & 0.81 & 41 & 23 & 14.2 & 17.0 & 0.022 & 16.4 & 16.36 & -0.02 & 1.204 & -28.3 & Q & PG $1338+416$ \\
\hline 13 & 47 & 19.40 & 59 & 2 & 32.5 & 10.3 & 0.025 & 16.2 & 16.48 & 0.22 & 0.768 & -27.3 & Q & SBS $1345+592$ \\
\hline 13 & 47 & 37.45 & 30 & 12 & 52.4 & 20.5 & 0.035 & 15.7 & 16.12 & 0.68 & 0.118 & -23.6 & $\mathrm{Q}$ & Q J1347+3012 \\
\hline 13 & 51 & 28.30 & 1 & 3 & 38.5 & 17.5 & 0.040 & 16.5 & 17.35 & 0.58 & 1.086 & -27.9 & Q & Q 1348+0118 \\
\hline 14 & 2 & 44.52 & 15 & 59 & 56.2 & 1.9 & 0.031 & 16.3 & 16.66 & 1.56 & 0.245 & -24.5 & BL & MC $1400+162$ \\
\hline 14 & 27 & 35.60 & 26 & 32 & 14.5 & 26.8 & 0.040 & 15.4 & 15.18 & -0.72 & 0.366 & -26.6 & Q & PG 1425+267 \\
\hline 14 & 36 & 45.79 & 63 & 36 & 37.6 & 32.1 & 0.038 & 15.7 & 16.61 & -0.22 & 2.066 & -30.4 & $\mathrm{Q}$ & S4 1435+63 \\
\hline 14 & 50 & 26.68 & 58 & 39 & 44.7 & 9.9 & 0.046 & 15.8 & 16.13 & 1.14 & 0.210 & -23.8 & $\mathrm{Q}$ & Mark 830 \\
\hline 14 & 51 & 53.63 & 72 & 14 & 46.8 & 5.8 & 0.027 & 16.3 & 16.96 & 0.16 & 0.75 & -27.1 & $\mathrm{Q}$ & HS $1451+7227$ \\
\hline
\end{tabular}


M.-P. Véron-Cetty et al.: Bright AGN in the ROSAT-FSC, Online Material p 7

Table 3. continued.

\begin{tabular}{|c|c|c|c|c|c|c|c|c|c|c|c|c|c|c|}
\hline \multicolumn{15}{|c|}{ J2000 USNO position } \\
\hline $\mathrm{h}$ & $\mathrm{m}$ & $\mathrm{S}$ & 2 & & "1 & $"$ & $c / r$ & $\overline{O_{U S}}$ & $O_{\mathrm{APS}}$ & $O-E$ & $z$ & $M_{\mathrm{abs}}$ & & Name \\
\hline$\overline{15}$ & 27 & 28.64 & 65 & 48 & 10.3 & 14.0 & 0.046 & 16.2 & 16.77 & 0.56 & 0.345 & -25.5 & $\mathrm{Q}$ & FBS $1526+659$ \\
\hline 15 & 50 & 43.65 & 11 & 20 & 47.4 & 23.7 & 0.040 & 16.3 & 16.33 & 0.14 & 0.436 & -25.9 & Q & MC $1548+114$ \\
\hline 15 & 51 & 58.16 & 58 & 6 & 44.7 & 4.5 & 0.036 & 16.2 & 16.74 & 1.14 & 1.320 & -28.7 & Q & SBS $1550+582$ \\
\hline 16 & 19 & 40.54 & 25 & 43 & 23.3 & 13.1 & 0.035 & 16.4 & 16.62 & 1.36 & 0.268 & -24.7 & Q & RX J16196+2543 \\
\hline 16 & 23 & 19.93 & 41 & 17 & 2.7 & 26.3 & 0.030 & 16.5 & 16.88 & 0.32 & 1.618 & -28.9 & Q & KUV $16217+4124$ \\
\hline 16 & 26 & 37.28 & 58 & 9 & 17.2 & 6.0 & 0.044 & 16.2 & 16.52 & 0.83 & 0.751 & -27.2 & Q & SBS $1625+582$ \\
\hline 16 & 28 & 25.70 & 8 & 33 & 0.1 & 10.1 & 0.031 & 16.4 & 17.22 & 0.32 & 0.44 & -25.8 & $\mathrm{Q}$ & HS $1626+0839$ \\
\hline 16 & 31 & 43.83 & 52 & 53 & 43.8 & 5.7 & 0.048 & 16.5 & 16.81 & 0.44 & 0.352 & -25.2 & Q & SBS $1630+530$ \\
\hline 16 & 32 & 1.11 & 37 & 37 & 50.0 & 14.5 & 0.034 & 16.0 & 16.60 & 0.54 & 1.478 & -29.4 & Q & PG 1630+377 \\
\hline 16 & 32 & 34.68 & 73 & 59 & 43.0 & 3.2 & 0.030 & 16.1 & 17.97 & 1.30 & 0.208 & -24.4 & Q & RXS J16325+7359 \\
\hline 16 & 34 & 29.01 & 70 & 31 & 32.3 & 1.2 & 0.049 & 14.9 & 15.26 & 1.03 & 1.337 & -30.1 & Q & PG 1634+706 \\
\hline 16 & 37 & 7.53 & 41 & 40 & 26.9 & 17.0 & 0.028 & 16.2 & 16.28 & 1.60 & 0.765 & -27.3 & Q & KUV $16355+4146$ \\
\hline 17 & 1 & 0.62 & 64 & 12 & 9.3 & 19.0 & 0.035 & 15.9 & 16.03 & 0.18 & 2.736 & -31.2 & Q & HS $1700+6416$ \\
\hline 17 & 4 & 41.39 & 60 & 44 & 30.5 & 8.4 & 0.042 & 15.6 & 15.86 & 0.76 & 0.371 & -26.3 & Q & 3C 351.0 \\
\hline 17 & 6 & 48.06 & 32 & 14 & 22.9 & 17.0 & 0.029 & 16.2 & 16.94 & 0.66 & 1.070 & -28.2 & Q & RGB J1706+322 \\
\hline 17 & 19 & 34.18 & 25 & 10 & 58.6 & 16.9 & 0.039 & 16.4 & 16.87 & 1.34 & 0.579 & -26.4 & Q & RX J17195+2510 \\
\hline 22 & 53 & 7.37 & 19 & 42 & 34.7 & 21.5 & 0.039 & 16.4 & 16.75 & 0.92 & 0.284 & -24.9 & Q & HS 2250+1926 \\
\hline 23 & 7 & 45.62 & 19 & 1 & 20.8 & 11.6 & 0.033 & 16.4 & 17.13 & 0.98 & 0.313 & -25.0 & Q & PKS 2305+18 \\
\hline 23 & 11 & 59.50 & 9 & 26 & 1.4 & 7.4 & 0.048 & 16.3 & 17.51 & 0.16 & 0.479 & -26.1 & Q & RX J23119+0925 \\
\hline 23 & 50 & 10.07 & 8 & 12 & 55.3 & 16.6 & 0.025 & 16.4 & 18.19 & 1.00 & 1.70 & -29.1 & Q & HS $2347+0756$ \\
\hline
\end{tabular}


Table 4. List of 103 QSO candidates identified with a ROSAT-FSC source and located in zone IVa. Column 1: right ascension, Col. 2: declination, Col. 3: separation between the ROSAT and USNO positions in arcsec, Col. 4: X-ray count rate (count $\mathrm{s}^{-1}$ ), Col. 5: magnitude $O_{\mathrm{USNO}}$, Col. 6: magnitude $O_{\mathrm{APS}}$, Col. 7: APS $O-E$ colour, Col. 8: redshift, Col. 9: absolute $O_{\mathrm{USNO}}$ magnitude, Col. 10: classification: DA, DN, WD: white dwarfs; *: stars; CV: cataclysmic variable; BL: BLLac object; S1: Seyfert 1 galaxy; S1n: Narrow Line Seyfert 1; Q: QSO; Col. 11: reference: (1) OAGH and (2) OHP observations, Col. 12: name.

\begin{tabular}{|c|c|c|c|c|c|c|c|c|c|c|c|c|c|c|c|}
\hline \multicolumn{16}{|c|}{ J2000 USNO position } \\
\hline $\mathrm{h}$ & $\mathrm{m}$ & $\mathrm{s}$ & & & & & $c / r$ & $O_{\mathrm{US}}$ & $O_{\mathrm{APS}}$ & $O-E$ & $z$ & $M_{\mathrm{abs}}$ & & Ref. & Name \\
\hline 0 & 11 & 19.46 & 28 & 17 & 50.8 & 18.7 & 0.023 & 15.5 & 15.88 & 0.12 & & & * & 1 & \\
\hline 0 & 13 & 4.80 & 10 & 11 & 28.6 & 5.8 & 0.023 & 16.0 & 16.25 & 0.32 & 0.239 & -24.9 & Q & 1 & \\
\hline 0 & 40 & 46.17 & 18 & 54 & 24.3 & 17.0 & 0.026 & 16.0 & 16.71 & -0.12 & 0.153 & -23.3 & Q & 1 & \\
\hline 1 & 7 & 37.51 & 22 & 22 & 32.3 & 33.7 & 0.042 & 16.2 & 16.89 & 0.68 & & & $*$ & 2 & \\
\hline 1 & 20 & 46.96 & 23 & 31 & 40.1 & 28.7 & 0.047 & 15.9 & 16.27 & 0.54 & & & $*$ & 2 & \\
\hline 1 & 37 & 37.24 & 30 & 2 & 49.1 & 13.1 & 0.035 & 13.5 & 16.90 & 0.36 & & & DN & & TX Tri \\
\hline 2 & 55 & 30.70 & 7 & 25 & 57.1 & 19.4 & 0.030 & 16.3 & 16.97 & 0.66 & 0.707 & -27.0 & Q & 1 & \\
\hline 3 & 53 & 15.60 & 9 & 56 & 35.1 & 24.0 & 0.030 & 16.0 & 16.49 & -0.82 & & & * & 2 & \\
\hline 8 & 23 & 46.65 & 24 & 53 & 51.2 & 18.5 & 0.039 & 15.6 & 15.83 & -0.40 & & & WD & 1 & TON 316 \\
\hline 8 & 39 & 34.28 & 23 & 34 & 10.4 & 12.8 & 0.041 & 15.8 & 16.49 & -0.52 & & & DA & & PG $0836+237$ \\
\hline 8 & 45 & 51.14 & 60 & 9 & 13.7 & 13.3 & 0.027 & 16.0 & 16.30 & -0.46 & & & DA & & PG $0841+603$ \\
\hline 8 & 50 & 20.09 & 54 & 33 & 50.5 & 17.3 & 0.038 & 16.4 & 16.78 & 0.62 & & & $*$ & 1 & \\
\hline 8 & 56 & 32.44 & 50 & 41 & 14.1 & 5.1 & 0.027 & 15.8 & 15.57 & -0.58 & 0.235 & -25.0 & Q & 1 & \\
\hline 9 & 3 & 32.62 & 16 & 26 & 1.4 & 21.7 & 0.037 & 16.2 & 16.59 & 0.24 & & & $*$ & 1 & LB 9181 \\
\hline 9 & 10 & 35.38 & 31 & 27 & 45.9 & 27.0 & 0.048 & 16.3 & 16.21 & 0.42 & & & * & 1 & \\
\hline 9 & 10 & 37.97 & 53 & 12 & 2.8 & 19.3 & 0.030 & 16.4 & 16.82 & 0.52 & & & $*$ & 1 & \\
\hline 9 & 12 & 15.50 & 1 & 19 & 59.3 & 3.8 & 0.041 & 16.5 & 16.76 & -0.50 & & & * & 1 & \\
\hline 9 & 20 & 47.61 & 51 & 25 & 38.8 & 33.1 & 0.021 & 15.7 & 15.65 & 0.12 & & & $*$ & 1 & \\
\hline 9 & 30 & 6.76 & 52 & 28 & 4.0 & 12.0 & 0.039 & 15.8 & 16.37 & -0.02 & & & $*$ & & PG 0926+527 \\
\hline 9 & 30 & 21.87 & 23 & 53 & 30.1 & 19.2 & 0.027 & 16.0 & 16.70 & 0.14 & 0.243 & -24.3 & $\mathrm{Q}$ & 1 & \\
\hline 9 & 30 & 34.46 & 20 & 44 & 16.9 & 5.2 & 0.045 & 16.1 & 17.09 & 0.64 & 1.169 & -28.4 & Q & 1 & \\
\hline 9 & 45 & 13.97 & 15 & 10 & 11.7 & 17.9 & 0.057 & 15.8 & 17.66 & 0.86 & & & $*$ & 1 & \\
\hline 9 & 46 & 34.50 & 13 & 50 & 58.3 & 34.4 & 0.027 & 15.8 & 16.75 & 0.10 & & & $\mathrm{CV}$ & 1 & \\
\hline 9 & 49 & 39.77 & 17 & 52 & 49.5 & 12.0 & 0.021 & 16.3 & 18.65 & 0.60 & & & & & \\
\hline 9 & 52 & 45.70 & 2 & 9 & 38.7 & 17.1 & 0.025 & 15.2 & 16.32 & -0.76 & & & DA & & PG 0950+024 \\
\hline 9 & 56 & 49.88 & 29 & 50 & 14.6 & 10.7 & 0.046 & 15.4 & 16.09 & -0.10 & 0.845 & -28.5 & Q & 1 & TON 465 \\
\hline 9 & 57 & 11.78 & 63 & 10 & 10.2 & 3.8 & 0.024 & 16.4 & 16.25 & -0.06 & 0.918 & -27.6 & Q & 1 & \\
\hline 10 & 6 & 14.64 & 44 & 19 & 7.1 & 15.6 & 0.030 & 16.5 & 16.93 & -0.30 & & & DA & & \\
\hline 10 & 34 & 53.07 & 44 & 57 & 23.1 & 20.5 & 0.023 & 16.1 & 16.57 & 0.38 & 1.422 & -29.0 & Q & 1 & \\
\hline 10 & 35 & 27.50 & 49 & 58 & 27.7 & 22.8 & 0.021 & 16.5 & 17.44 & 0.36 & 1.427 & -28.5 & Q & 1 & \\
\hline 10 & 44 & 19.33 & 19 & 57 & 47.9 & 24.2 & 0.039 & 15.6 & 15.90 & 0.44 & & & $*$ & 1 & \\
\hline 10 & 46 & 42.30 & 39 & 20 & 18.2 & 8.3 & 0.043 & 16.4 & 17.16 & 0.22 & 0.390 & -25.3 & Q & 1 & \\
\hline 10 & 47 & 30.55 & 10 & 17 & 28.9 & 17.7 & 0.228 & 15.4 & 15.81 & 0.08 & 0.145 & -24.3 & Q & 1 & \\
\hline 11 & 8 & 42.46 & 16 & 50 & 40.2 & 31.2 & 0.030 & 15.6 & 16.48 & 0.04 & & & $*$ & 1 & \\
\hline 11 & 14 & 1.95 & 52 & 27 & 11.6 & 29.8 & 0.023 & 15.5 & 15.62 & 0.40 & & & $*$ & 1 & \\
\hline 11 & 15 & 7.70 & 2 & 37 & 57.7 & 7.6 & 0.024 & 16.5 & 17.08 & -0.04 & 0.564 & -26.1 & Q & 1 & \\
\hline 11 & 26 & 16.19 & 32 & 59 & 53.7 & 29.3 & 0.029 & 16.2 & 15.66 & 0.24 & & & $*$ & 1 & \\
\hline 11 & 33 & 31.23 & 58 & 57 & 47.8 & 19.9 & 0.025 & 15.9 & 15.98 & 0.44 & & & * & 1 & \\
\hline 11 & 34 & 25.08 & 23 & 16 & 8.8 & 24.0 & 0.039 & 16.3 & 16.66 & 0.64 & & & $*$ & 1 & \\
\hline 11 & 38 & 36.32 & 47 & 55 & 10.0 & 13.3 & 0.046 & 16.1 & 17.53 & -0.48 & & & * & 1 & \\
\hline 11 & 41 & 52.82 & 25 & 35 & 33.5 & 10.8 & 0.042 & 16.3 & 16.82 & -0.96 & & & * & 1 & \\
\hline 11 & 47 & 47.34 & 26 & 0 & 49.0 & 10.9 & 0.042 & 15.5 & 15.84 & 0.00 & & & * & 1 & \\
\hline 12 & 19 & 57.97 & 27 & 8 & 57.2 & 9.5 & 0.023 & 16.1 & 17.17 & 0.76 & & & * & 1 & \\
\hline 12 & 31 & 25.72 & 25 & 55 & 59.8 & 5.7 & 0.032 & 15.8 & 16.16 & 0.54 & & & * & 1 & \\
\hline 12 & 32 & 54.28 & 36 & 44 & 7.4 & 32.5 & 0.022 & 16.0 & 16.61 & -0.08 & & & WD & 1 & CBS 353 \\
\hline 12 & 52 & 30.85 & 14 & 26 & 9.3 & 24.3 & 0.029 & 16.3 & 17.30 & 0.10 & & & & & \\
\hline 12 & 57 & 37.02 & 16 & 30 & 48.6 & 13.5 & 0.035 & 16.1 & 16.95 & 0.66 & 1.017 & -28.2 & Q & 1 & \\
\hline 12 & 59 & 38.22 & 60 & 38 & 59.4 & 15.9 & 0.039 & 16.3 & 16.47 & -0.48 & & & $*$ & 1 & SBS $1257+609$ \\
\hline 12 & 59 & 44.45 & 68 & 4 & 0.8 & 30.9 & 0.022 & 16.3 & 16.64 & -0.72 & & & $*$ & 1 & FBS $1257+683$ \\
\hline 13 & 0 & 6.40 & 44 & 42 & 50.9 & 13.9 & 0.036 & 16.2 & 16.06 & 0.46 & & & * & 1 & \\
\hline 13 & 10 & 11.30 & 7 & 58 & 16.5 & 21.2 & 0.030 & 15.8 & 16.52 & -0.08 & 0.578 & -27.0 & Q & 1 & \\
\hline 13 & 13 & 15.90 & 9 & 18 & 20.4 & 27.7 & 0.021 & 16.4 & 17.34 & 0.10 & 1.790 & -29.2 & Q & 1 & \\
\hline 13 & 14 & 30.78 & 13 & 7 & 45.8 & 23.8 & 0.044 & 16.3 & 17.00 & 0.24 & 0.741 & -27.1 & Q & 1 & \\
\hline 13 & 20 & 1.10 & 7 & 18 & 17.1 & 21.1 & 0.038 & 16.5 & 16.97 & 0.74 & 0.866 & -26.9 & $\mathrm{Q}$ & 1 & \\
\hline 13 & 20 & 22.53 & 30 & 56 & 22.4 & 19.8 & 0.029 & 16.4 & 17.22 & -0.04 & 1.587 & -28.1 & Q & 1 & US 583 \\
\hline 13 & 24 & 47.70 & 3 & 24 & 32.9 & 15.5 & 0.040 & 16.5 & 16.84 & 0.54 & 0.303 & -24.9 & Q & 1 & \\
\hline
\end{tabular}


Table 4. continued.

\begin{tabular}{|c|c|c|c|c|c|c|c|c|c|c|c|c|c|c|c|}
\hline \multicolumn{16}{|c|}{ J2000 USNO position } \\
\hline $\mathrm{h}$ & $\mathrm{m}$ & $\mathrm{s}$ & 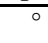 & & " & " & $c / r$ & $O_{U S}$ & $O_{\mathrm{APS}}$ & $O-E$ & $z$ & $M_{\mathrm{abs}}$ & & Ref. & Name \\
\hline 13 & 32 & 51.09 & 15 & 29 & 30.9 & 23.9 & 0.042 & 15.5 & 15.56 & 0.14 & & & * & 1 & \\
\hline 13 & 33 & 45.80 & 5 & 38 & 42.6 & 22.2 & 0.028 & 15.5 & 15.74 & 0.10 & & & $*$ & 1 & \\
\hline 13 & 40 & 59.95 & 60 & 26 & 11.8 & 17.6 & 0.034 & 16.2 & 16.48 & -0.68 & & & DA & & SBS 1339+606 \\
\hline 13 & 42 & 46.90 & 18 & 44 & 43.8 & 22.4 & 0.039 & 16.4 & 16.69 & 0.12 & 0.382 & -25.5 & $\mathrm{Q}$ & 1 & \\
\hline 13 & 50 & 13.97 & 14 & 35 & 47.4 & 30.4 & 0.023 & 16.4 & 16.93 & 0.48 & & & $*$ & 1 & \\
\hline 13 & 51 & 25.25 & 19 & 5 & 33.4 & 10.8 & 0.027 & 16.4 & 16.89 & 0.64 & & & * & 1 & \\
\hline 13 & 54 & 48.82 & 49 & 13 & 37.1 & 19.8 & 0.028 & 15.5 & 15.68 & 0.32 & & & * & 1 & \\
\hline 13 & 55 & 43.99 & 20 & 12 & 31.8 & 19.9 & 0.030 & 15.6 & 16.11 & 0.30 & & & * & 1 & \\
\hline 13 & 58 & 41.50 & 2 & 49 & 12.5 & 21.1 & 0.046 & 16.2 & 16.85 & -0.08 & & & * & 1 & \\
\hline 14 & 6 & 58.74 & 14 & 42 & 38.6 & 13.0 & 0.034 & 15.4 & 15.84 & 0.34 & 0.264 & -25.7 & Q & 1 & \\
\hline 14 & 9 & 39.24 & 28 & 16 & 49.9 & 25.2 & 0.035 & 16.1 & 16.93 & 0.34 & 0.165 & -23.9 & $\mathrm{Q}$ & 1 & \\
\hline 14 & 10 & 57.74 & 64 & 33 & 10.6 & 18.8 & 0.041 & 16.4 & 16.83 & 0.06 & 0.462 & -25.9 & Q & 1 & \\
\hline 14 & 17 & 30.16 & 13 & 0 & 1.6 & 11.6 & 0.021 & 15.7 & 16.00 & 0.38 & & & $*$ & 1 & \\
\hline 14 & 19 & 25.77 & 38 & 2 & 49.0 & 12.7 & 0.039 & 16.2 & 17.22 & 0.08 & 0.517 & -26.3 & Q & 1 & \\
\hline 14 & 31 & 10.98 & 14 & 23 & 8.3 & 3.7 & 0.022 & 16.1 & 15.91 & -0.04 & 1.425 & -29.0 & Q & 1 & \\
\hline 14 & 47 & 50.12 & 38 & 5 & 30.3 & 12.2 & 0.023 & 15.8 & 16.28 & 0.04 & & & $*$ & 1 & \\
\hline 15 & 0 & 31.80 & 48 & 36 & 47.0 & 16.0 & 0.021 & 16.4 & 16.72 & 0.48 & 1.031 & -27.9 & Q & 1 & \\
\hline 15 & 5 & 27.62 & 29 & 47 & 18.7 & 8.7 & 0.036 & 15.0 & 15.65 & -0.34 & 0.527 & -27.6 & $\mathrm{Q}$ & 1 & CSO 1080 \\
\hline 15 & 8 & 32.28 & 67 & 42 & 43.4 & 9.0 & 0.032 & 16.0 & 17.12 & 0.14 & 0.336 & -25.8 & Q & 1 & \\
\hline 15 & 12 & 44.60 & 9 & 31 & 0.8 & 23.0 & 0.037 & 15.9 & 16.61 & 0.56 & & & $*$ & 1 & \\
\hline 15 & 16 & 32.30 & 12 & 13 & 50.5 & 10.6 & 0.028 & 16.2 & 17.26 & 0.78 & & & BL & 1 & \\
\hline 15 & 44 & 3.76 & 26 & 48 & 38.6 & 21.7 & 0.023 & 16.4 & 17.15 & 0.74 & & & $*$ & 1 & \\
\hline 15 & 45 & 53.50 & 9 & 36 & 20.6 & 19.6 & 0.032 & 16.1 & 16.40 & -0.22 & & & & & \\
\hline 15 & 48 & 33.03 & 44 & 22 & 26.1 & 12.7 & 0.021 & 16.3 & 16.97 & 0.48 & 0.322 & -25.2 & Q & 1 & \\
\hline 15 & 51 & 9.60 & 45 & 42 & 52.1 & 22.5 & 0.036 & 15.6 & 15.95 & 0.32 & & & & & \\
\hline 15 & 51 & 52.45 & 19 & 11 & 4.1 & 34.9 & 0.034 & 15.8 & 16.31 & 0.50 & & & & & \\
\hline 15 & 53 & 4.93 & 35 & 48 & 28.6 & 25.2 & 0.033 & 16.1 & 17.20 & 0.72 & 0.722 & -26.8 & Q & 1 & \\
\hline 15 & 56 & 9.90 & 3 & 9 & 22.5 & 9.6 & 0.037 & 16.3 & 16.80 & 0.48 & 0.131 & -23.2 & S1n & 1 & \\
\hline 16 & 5 & 19.72 & 14 & 48 & 52.5 & 15.5 & 0.040 & 16.2 & 16.51 & 0.00 & 0.371 & -25.7 & Q & 1 & \\
\hline 16 & 9 & 47.80 & 7 & 12 & 33.2 & 27.8 & 0.021 & 16.4 & 16.95 & 0.64 & & & * & 1 & \\
\hline 16 & 11 & 36.58 & 15 & 20 & 54.6 & 18.8 & 0.030 & 16.3 & 16.69 & 0.40 & 1.309 & -28.6 & Q & 1 & \\
\hline 16 & 17 & 42.10 & 6 & 2 & 23.8 & 20.4 & 0.078 & 15.5 & 15.69 & 0.42 & & & $*$ & 1 & \\
\hline 16 & 28 & 35.38 & 45 & 20 & 43.3 & 34.1 & 0.022 & 16.4 & 17.00 & 0.68 & & & $*$ & 1 & \\
\hline 16 & 30 & 9.65 & 45 & 16 & 37.9 & 21.7 & 0.022 & 16.5 & 16.96 & 0.32 & & & $*$ & 1 & \\
\hline 16 & 31 & 10.46 & 38 & 44 & 49.6 & 7.8 & 0.036 & 15.8 & 15.97 & 0.48 & & & $*$ & 1 & \\
\hline 16 & 45 & 20.15 & 61 & 35 & 9.7 & 3.8 & 0.023 & 16.4 & 16.89 & 0.64 & 0.410 & -25.5 & $\mathrm{Q}$ & 1 & \\
\hline 16 & 46 & 15.52 & 25 & 41 & 43.3 & 15.7 & 0.047 & 16.4 & 17.26 & 0.56 & 0.188 & -23.9 & Q & 1 & \\
\hline 17 & 18 & 28.97 & 57 & 34 & 22.3 & 6.4 & 0.039 & 15.9 & 16.07 & 0.48 & 0.100 & -22.9 & S1n & 1 & \\
\hline 17 & 20 & 13.19 & 49 & 55 & 26.4 & 28.8 & 0.027 & 16.0 & 15.89 & 0.36 & & & $*$ & 1 & \\
\hline 17 & 21 & 45.75 & 57 & 16 & 56.7 & 31.5 & 0.021 & 15.8 & 15.87 & 0.24 & & & $*$ & 1 & \\
\hline 17 & 28 & 13.44 & 32 & 22 & 5.8 & 10.8 & 0.022 & 16.4 & 17.08 & 0.34 & 0.563 & -26.3 & Q & 1 & \\
\hline 17 & 29 & 35.53 & 52 & 30 & 47.5 & 3.0 & 0.029 & 16.2 & 15.96 & 0.02 & 0.278 & -25.0 & Q & 1 & \\
\hline 17 & 49 & 12.30 & 55 & 12 & 8.9 & 28.7 & 0.023 & 16.3 & 16.58 & 0.44 & & & WD & 1 & \\
\hline 22 & 37 & 31.84 & 10 & 19 & 4.0 & 3.8 & 0.024 & 16.5 & 16.77 & -0.46 & 0.103 & -22.5 & S1 & 1 & \\
\hline 22 & 37 & 33.28 & 10 & 18 & 42.7 & 26.3 & 0.024 & 15.9 & 16.10 & 0.50 & & & * & 1 & \\
\hline 23 & 7 & 13.30 & 4 & 32 & 2.5 & 29.8 & 0.079 & 15.9 & 17.25 & -0.92 & & & $*$ & 1 & \\
\hline 23 & 15 & 52.73 & 11 & 33 & 2.1 & 21.4 & 0.036 & 16.5 & 17.20 & 0.24 & 0.567 & -26.2 & Q & 1 & KUV 23134+1117 \\
\hline
\end{tabular}

